\title{
Causal Nexus between Financial Integration and Economic Growth : Does Nonlinearity Matter?
}

\author{
Sami Saafi \\ EAS - Faculty of Economic Sciences and Management of Mahdia University of Monastir, Tunisia \\ Meriem Bel Haj Mohamed \\ EAS - Faculty of Economic Sciences and Management of Mahdia University of Monastir, Tunisia \\ Makram Ben Doudou \\ EAS - Faculty of Economic Sciences and Management of Mahdia University of Monastir, Tunisia
}

\begin{abstract}
Empirical studies that rely on a linear framework typically fail to find evidence of a causal link between financial integration and economic growth. In this study, we extend the analysis by applying both linear and nonlinear Granger-causality tests to data for 19 emerging and developing countries. Consistent with previous research, the linear causality analysis reveals only weak causal linkages between financial integration and economic growth. In contrast, the nonlinear causality analysis provides evidence of significant nonlinear causality in 18 out of 19 countries. The growth hypothesis holds true for Argentina, Bolivia, Colombia, Morocco, Tunisia, and Venezuela whereas a reverse relation was found in Brazil, Chile, Cote d'Ivoire, Costa Rica, Ecuador, Egypt, South Korea, Malaysia, Mexico, and Paraguay. The feedback hypothesis also exists in Bolivia and Uruguay. Overall, the divergent results in the 19 countries imply that

\footnotetext{
* Corresponding Author: Sami Saafi; EAS - Faculty of Economic Sciences and Management of Mahdia, Mahdia Hiboun Cité Sidi Messaoud, Mahdia 5111, Tunisia; Tel: +216 73683 191, Fax:+216 73683 190, E-mail: samisaafi@yahoo.fr.

Co-authors: Meriem Bel Haj Mohamed; EAS - Faculty of Economic Sciences and Management of Mahdia, Mahdia Hiboun Cité Sidi Messaoud, Mahdia 5111, Tunisia; Tel: +216 73683 191, Fax:+216 73683 190, E-mail:meriem.hjmed@yahoo.fr.

Makram Ben Doudou; EAS - Faculty of Economic Sciences and Management of Mahdia, Mahdia Hiboun Cité Sidi Messaoud, Mahdia 5111, Tunisia; Tel: +216 73683 191, Fax:+216 73683 190, E-mail:m_bendoudou@yahoo.fr.
} 
policies cannot be uniformly implemented as there would have been different effects in each country.

JEL Classifications: C14, C22, F3, O4

Keywords: Financial Integration, Economic Growth, Nonlinear Granger Causality, Developing Countries, Emerging Economies

\section{Introduction}

Over the past three decades, there has been a rapid and intense global financial integration process, especially for emerging and developing economies (Lane and Milesi-Ferretti 2007, Vo and Daly 2007, Gehringer 2015, Malik 2015, Ahmed 2016). From a theoretical viewpoint, such financial integration should promote international consumption risk-sharing and, thereby, enhance production specialization, capital allocation, and economic growth (Obstfeld 1994, Acemoglu and Zilibotti 1997). Financial integration may also spur economic growth indirectly through improved factor productivity. This occurs, as noted by Gehringer (2013), via better and more efficient allocation of resources (Obstfeld 1994, Acemoglu and Zilibotti 1997, Edison et al. 2002), as well as easier access to investment opportunities (Giannett et al. 2002). Moreover, financial openness may enhance the functioning of domestic financial systems through intensification of competition and the import of financial services (Klein and Olivei 2001, Levine 2001). This in turn leads to greater investment and, hence, faster economic growth.

The nexus between financial integration and economic growth has been closely analyzed by a number of studies, but the empirical evidence in these studies remains controversial and ambiguous. While some support the view that capital account liberalization has a positive effect on economic growth (Quinn 1997, Henry 2000, Bekaert et al. 2005, Klein and Olivei 2008, Quinn and Toyoda 2008, Vithessonthi and Tongurai 2012, De Nicolò and Juvenal 2014), others fail to provide such support (Grilli and Milesi-Ferretti 1995, Kraay 1998, Rodrick 1998, Edison et al. 2002, Fratzscher and Bussière 2004, Bumann et al. 2013 among others). A few other empirical researchers also report evidence of a negative effect of financial liberalization on growth (Eichengreen 
and Leblang 2003, Ahmed 2011, Ahmed 2016). These mixed results have been attributed to, among other elements, the limitations of the empirical approaches used. One major problem with the cross-country approach commonly employed in the aforementioned studies is its inability to capture country- and economy-specific experiences in terms of growth dynamics (Mmolainyane and Ahmed 2015). In other words, the presence of a significant relation in some countries does not necessarily imply that this exists in other countries as well. Such heterogeneity across countries is due to differences in financial and asset characteristics, institutional setup, and levels of economic development (Ahmed 2013). These differences suggest that the financial integration-growth relation may be country-specific. Therefore, it is necessary to recognize the heterogeneous nature of the countries under investigation.

In recognition of this situation, in a newly emerging strand of literature, researchers have increasingly turned to time-series analysis that enables them to control for the presence of country-specific heterogeneity and cope with the endogeneity problem and/ or causal mechanisms. However, these times-series studies also have mixed results. For instance, Ray (2012) examined the relation between financial integration and economic growth in India for the 1990 2010 period, using the Johansen procedure (Johansen 1988, Johansen and Juselius 1990). The study's empirical evidence reported a unidirectional causality running from economic growth to financial integration with no feedback. Hye and Wizarat (2013) analyzed the financial liberalization-growth nexus in Pakistan for the 1971 2007 period. The authors adopted the Autoregressive Distributed lag (ADL) approach to cointegration for their empirical analysis. Their findings indicated no significant long-run impact of financial integration on growth.

In an extensive study, Ahmed and Mmolainyane (2014) examined the long-run equilibrium and causal relations between financial integration and economic growth in Botswana. Their study was based on a production function framework, controlling for trade openness, physical capital, labor, inflation, and institutional structure. Using a Vector Error Correction Model (VECM) and annual data for the 1974 2009 period, they found that financial integration had no significant direct effect on economic growth but did play an indirect role in growth through positive effects on financial development. The authors explained these results by the weakness in Foreign Direct Investment (FDI) inflows to this country during the sample period. In a more recent study, Dinar et al. (2015) investigated the relation between financial liberalization and economic growth in Turkey for 1998 2012 period using Gregory and Hansen's (1996) testing approach for threshold cointegration. They found a long-run cointegrating relation among the 
variables. To achieve these results, the authors also carried out Toda and Yamamoto's (1995) causality procedure and found evidence of a unidirectional Granger causality running from economic growth to financial liberalization.

As the above literature review indicates, most empirical studies dealing with causality between financial integration and economic growth resort only to traditional linear Granger causality tests. This means that researchers often neglect a possible nonlinear relation between these variables because the traditional Granger causality test, designed to detect linear causality, is ineffective in uncovering certain nonlinear relations (Baek and Brock 1992, Hiemstra and Jones 1994). Recent empirical evidence, however, suggests that this relation is very likely to be nonlinear in that the growth effect of financial integration may vary under alternative economic or financial conditions (Kose et al. 2011, Friedrich et al. 2013, Malik 2015). In a number of earlier empirical studies, this type of nonlinear behavior has been parsimoniously captured by panel threshold regression models (Chen and Quang 2014). Nevertheless, these studies adopt a unidirectional approach, ignoring the possibility of mutual dependence between financial integration and economic growth. Therefore, this study aims to fill this literature gap by applying linear and nonlinear Granger causality tests in investigating the causality between the two variables studied. In particular, besides the linear Granger causality test of Toda and Yamamoto (1995), the nonlinear Granger test proposed by Kyrtsou and Labys (2006) is also applied to capture both linear and nonlinear Granger causality between financial integration and economic growth.

The examination of nonlinear and asymmetric causal links between financial integration and economic growth is motivated by both theoretical and empirical insights. Indeed, most economic and financial time series exhibit a nonlinear behavior over time and tend to interact with each other in a nonlinear fashion. This recognition has been confirmed by, among others, the occurrences of severe economic and financial crises (e.g., the 1997 1998 Asian financial crisis, the 2007 2008 US subprime crisis, and the 2008 2009 global financial crisis), wars and other extreme events(e.g., the September 11, 2001 terrorist attack, the Second Gulf war in 2003, the 2006 oil price shock, and the Arab Spring movements), sudden changes in macroeconomic policies, financial and economic reforms, increased complexity of financial markets, structural change, and reallocation shocks. All the aforementioned factors may cause unexpected changes in the behavior of economic and financial variables, which particularly induce financial structural breaks, asymmetric responses to shocks, and leverage effects (Ajmi et al. 2013, Atil et al. 2014, Bildirici and Turkmen 2015). Under these circumstances, financial 
integration and economic growth are likely to exhibit a nonlinear pattern, and their joint dynamics imply a more complex than just a simple and stable relationship. In view of this, nonparametric analysis techniques are more suitable because they place direct emphasis on prediction without imposing a linear functional form (Saafi et al. 2015a). The failure in previous literature to account for asymmetry and nonlinearity between financial integration and economic growth may have resulted in incorrect inferences about the existence/non-existence of the financial integration-growth relation.

This research aims to examine whether there is a nonlinear causal relationship in the financial integration-growth nexus in 19 emerging and developing countries for the 1970 2011 period. Specifically, this study makes three main contributions. First, it takes a novel approach in examining the countries under investigation, deviating from the common use in the literature of cross-country and panel regression analysis to the use of separate regression models for each country. Through this approach, we can control for any differences in the financial and economic environment across countries. Notwithstanding its significance, there has been limited research that has adopted country-specific time series data to examine the effect of financial integration on economic growth. Second, this study considers three types of financial integration indicators to quantify the impact of financial openness on growth and, further, to examine the sensitivity of the results. Third, unlike previous time-series studies that have investigated the link between financial integration and economic growth, we do not assume that the dynamics of this relationship are linear. In addition to the linear Granger causality test, we employ Kyrtsou and Labys 's nonlinear method, which enables us to test for nonlinear Granger causality and, at the same time, avoid making spurious inferences. To the best of our knowledge, this is the first study to employ the nonlinear causality test of Kyrtsou and Labys (2006) based on the bivariate noisy Mackey-Glass (hereafter $\mathrm{M}-\mathrm{G}$ ) process to explore the nonlinear relation between financial integration and economic growth. The most important feature of the nonlinear $\mathrm{M}-\mathrm{G}$ process is that it enables the filtering of the more complex dependent dynamics in a time series (Kyrtsou and Labys 2006). Because of this advantage, it has been widely used in the literature (Kyrtsou and Labys 2006, Hristu Varsekelis and Kyrtsou 2008, Kumar 2009, Kumar and Thenmozhi 2012, Ajmi et al. 2013, Bildirici and Turkmen 2015, Saafi et al. 2015a, 2015b). It is expected that the analysis in this study will add new insights to the existing literature that will help the policymakers to formulate and implement sound economic policies in order to sustain economic development.

The remainder of the study is structured as follows. Section II describes the empirical 
methodology. Section III discusses the data and presents some summary statistics. Section IV reports the empirical results, while Section V provides concluding remarks.

\section{Methodology}

In this study, we applied both linear and nonlinear Granger causality tests to explore the dynamic relations between financial integration and economic growth. The corresponding techniques, i.e., the linear and nonlinear Granger causality tests, are respectively described in subsections $\mathrm{A}$ and $\mathrm{B}$.

\section{A. Granger causality approach}

Following the pioneering contribution of Granger (1969), various versions of Granger causality tests have been proposed by researchers to examine the short-run causal relation between variables (Sims et al.1990, Toda and Phillips 1993, Toda and Yamamoto 1995, Dolado and Lutkepohl 1996). Among those, Toda and Yamamoto's non-causality test has attracted a great deal of interest over the years in both empirical and theoretical studies. One of its greatest advantages is that it does not require pre-testing for integration or cointegration properties of the Vector Auto-Regression (VAR) system and thus avoids the potential biases of pre-testing that undermine traditional causality tests (Rambaldi and Doran 1996 , Zapata and Rambaldi 1997, Clark and Mirza 2006). In other words, unlike the conventional Granger causality test, the Toda-Yamamoto technique fits a standard VAR on levels of the variables and not on their first differences, thereby minimizing the risks perhaps associated with misidentifying the orders of integration of the series or the presence of cointegration. In addition, it minimizes the possibility of distorting the test size, which frequently results from pre-testing (Giles 1997, Mavrotas and Kelly 2001).

The adoption of the Toda and Yamamoto (1995) approach to causality testing in empirical studies has become increasingly popular in the literature. This approach has also become a prominent technique to study the linear causal relation between economic and financial variables. For example, it has been shown to be especially useful for exploring the relations between healthcare expenditure and GDP (Amiri and Ventelou 
2012), oil and commodity prices (Nazlioglu and Soytas 2011), oil price shocks and stock market performance (Le and Chang 2015), terms of trade and economic growth (Jawaid and Raza 2013), energy consumption and output (Payne 2009, Menyah and Wolde-Rufael 2010), debt and growth (Kemba and Khan 2016), the shadow economy and unemployment (Saafi et al. 2015a, 2015b), and economic growth and financial development (Abu-Bader and Abu-Qarn 2008, Wolde-Rufael 2009).

Toda and Yamamoto (1995) used the modified Wald (MWALD) statistic for testing linear restrictions on the coefficients in an augmented VAR $\left(k+d_{\max }\right)$ model, where $\mathrm{k}$ is the optimal lag order in the VAR system and $d_{\max }$ is the maximal order of integration in the model. The MWALD statistic follows an asymptotic $x^{2}$ distribution with k degrees of freedom $\left(x^{2}(k)\right)$. Two steps are involved in implementing the procedure. In the first step, the optimal lag length $(k)$ and the maximum order of integration $\left(d_{\max }\right)$ of the series under consideration have to be determined using one of the information criteria methods. Such a step is crucial as it avoids spurious causality or absence of causality (Clark and Mirza 2006). The selected $\operatorname{VAR}(k)$ is then augmented by the maximal order of integration and a VAR of order $\left(k+d_{\max }\right)$ is estimated. In the second step, the modified Wald test is applied to the first $\mathrm{k}$ VAR coefficient matrix (but not all lagged coefficients) to conduct inference on Granger causality.

In accordance with that approach, the financial integration-economic growth model is represented with the following VAR system:

$$
\begin{aligned}
& E G_{t}=\beta_{0}+\sum_{i=1}^{k} \beta_{1 i} E G_{t-i}+\sum_{j=k+1}^{k+d_{\max }} \beta_{2 j} E G_{t-j}+\sum_{i=1}^{k} \phi_{1 i} F I_{t-i}+\sum_{j=k+1}^{k+d_{\max }} \phi_{2 j} F I_{t-j}+\varepsilon_{2 t} \\
& F I_{t}=\alpha_{0}+\sum_{i=1}^{k} \alpha_{1 i} F I_{t-i}+\sum_{j=k+1}^{k+d_{\max }} \alpha_{2 j} F I_{t-j}+\sum_{i=1}^{k} \gamma_{1 i} E G_{t-i}+\sum_{j=k+1}^{k+d_{\max }} \gamma_{2 j} E G_{t-j}+\varepsilon_{1 t}
\end{aligned}
$$

Where $F I$ denotes the financial integration indicator and $E G$ denotes the per-capita GDP growth rate (proxy for economic growth). $\varepsilon_{1 t}$ and $\varepsilon_{2 t}$ are error terms that are assumed to be white noise with zero mean, constant variance and no autocorrelation. From Equstion (1) Granger causality from $F I_{t}$ to $E G_{t}$ implies $\phi_{1 i} \neq 0 \forall i$; similarly in Equstion (2), $E G_{t}$ Granger-causes $F I_{t}$ if $\gamma_{1 i} \neq 0 \forall i$. For testing the null hypothesis, Toda and Yamamoto (1995) suggested that the conventional F-statistic used for the traditional Granger causality test may be invalid as the test does not have a standard distribution 
when the time series data are integrated or cointegrated. Zapata and Rambaldi (1997) used Monte Carlo simulations to investigate the size and power properties of three different versions of the Granger non-causality test in standard and modified form, including the MWALD test proposed by Toda and Yamamoto (1995). The results show that the MWALD test exhibits better power and size properties relative to the likelihood and WALD tests.

\section{B. Kyrtsou-Labys nonlinear Granger causality approach}

One of the common criticisms of the linear approach to causality testing is that such tests fail to detect nonlinear causal relations. Owing to this weakness, various nonparametric causality tests have been proposed in the literature. The earliest test is the one suggested by Baek and Brock (1992), which is based on the correlation integral, a measure of spatial dependence across time and is applied to the residuals of linear Granger causality models. One main shortcoming of this test is that it depends on the assumption that the variables are mutually independent and identically distributed (hereafter iid). This is relaxed in the study by Hiemstra and Jones (1994). They developed a modified test statistic for the nonlinear causality, which allows each series to exhibit short-term temporal dependence. To detect nonlinear causal relations, the modified Baek and Brock test is applied to the residual series from a VAR model and not to the initial stationary variables as input in the model. However, as pointed out by Kyrtsou and Labys (2006), linear filtering of data using VAR methodology before the application of the Hiemstra and Jones test of nonlinear Granger causality can lead to serious distortions. To overcome this drawback, Kyrtsou and Labys (2006) proposed a new test procedure which could be used to detect a possible nonlinear causality relation between two time series.

To define nonlinear Granger causality, Kyrtsou and Labys (2006) proposed a bivariate noisy $\mathrm{M}-\mathrm{G}$ model. Its general form is as follows:

$$
\begin{aligned}
& E G_{t}=\alpha_{11} \frac{E G_{t-\tau_{1}}}{1+E G_{t-\tau_{1}}^{c_{1}}}-\beta_{11} E G_{t-1}+\alpha_{12} \frac{F I_{t-\tau_{2}}}{1+F I_{t-1}^{c_{2}}}-\beta_{12} F I_{t-1}+\xi_{1, t} \\
& F I_{t}=\alpha_{21} \frac{E G_{t-\tau_{1}}}{1+E G_{t-\tau_{1}}^{c_{1}}}-\beta_{21} E G_{t-1}-\alpha_{22} \frac{F I_{t-\tau_{2}}}{1+F I_{t-1}^{c_{2}}}-\beta_{22} F I_{t-1}+\xi_{2, t}
\end{aligned}
$$


where $\xi_{2, t}$ and $\xi_{2, t}: N(0,1), t=\tau, \ldots, N, \tau=\max \left(\tau_{1}, \tau_{2}\right) \cdot \beta_{i j}$ and $\alpha_{i j}$ indicate the nonlinear and linear effects of the independent variables on the dependent variable, respectively. $\tau_{1}$ denotes the integer delays, and $c_{i}$ denotes the constants which can be chosen via prior selection. In this study, following Kyrtsou and Labys' suggestion, the best delays (lags), $\tau_{1}$ and $\tau_{2}$, are selected on the basis of likelihood ratio tests and the Schwarz criterion. The Kyrtsou and Labys' causality test is similar to the linear Granger causality test, except that the models fitted to the series are $M-G$ processes. This test is performed by estimating the $\mathrm{M}-\mathrm{G}$ model parameters under no constraint with ordinary least squares. To test reverse causality (i.e., from $F I$ to $E G$ ), another $\mathrm{M}-\mathrm{G}$ model is estimated under the constraint $\alpha_{12}=0$ that reflects our null hypothesis. Let $\hat{\vartheta}$ and $\hat{v}$ be the residuals obtained by the unconstrained and constrained best-fit $\mathrm{M}-\mathrm{G}$ model, respectively. Thus, the corresponding sums of residual squares can be written as $S_{c}=\sum_{t-1}^{T} \hat{v}^{2}$. and $S_{c}=\sum_{t-1}^{T} \hat{v}^{2}$. Let $n_{u}=4$ be the number of free parameters in the M-G model and on the other side $n_{c}=1$ be the number of parameters required to be zero when estimating the restricted model. Evidently, the test statistic satisfies the following:

$$
S_{F}=\frac{\left(S_{c}-S_{u}\right) / n_{c}}{S_{u} /\left(T-n_{u}-1\right)} \sim F\left(n_{c}, T-n_{u}-1\right),
$$

where $S_{F}$ is the test statistic.

\section{Data and Summary Statistics}

\section{A. Data sources}

The annual data used in this study cover the 1970 2011 for 19 emerging and developing countries-Argentina, Bolivia, Botswana, Brazil, Chile, Colombia, Cote d'Ivoire, Costa Rica, Ecuador, Egypt, Malaysia, Mexico, Morocco, Paraguay, Peru, South Korea, Tunisia, Uruguay, and Venezuela. The selection of countries and sample period is dictated by data availability, especially the availability of data on financial integration. Data on per capita GDP growth are sourced from the online World Bank's World Development Indicators database. As a measure of financial integration, 
following Bekaert et al. (2005), Honig (2008), Ahmed and Mmolainyane (2014), Motelle and Biekpe (2015), and Ahmed (2016), among others, we use the capital account openness index, developed by Chinn and Ito (2008). This indicator (KAOPEN) is the first principal component of the binary variables pertaining to cross-border financial transactions, based on the intensity of controls reported in the IMF's Annual Report on Exchange Arrangements and Exchange Restrictions (AREAER). For obtaining a more robust and appropriate measure of international financial integration, we also construct two de facto financial openness indicators using the updated and extended version of the External Wealth of Nations Mark II database compiled by Lane and Milesi-Ferretti (2007). The first indicator is the aggregate stock of external assets and liabilities to GDP (LMF1). The second measure of the extent of international financial integration is the sum of total foreign equity assets and liabilities as a share of GDP (LMF2). Table 1 provides the summary statistics of these variables. As can be seen in the table, there is a wide variation in per capita GDP growth among the countries. The mean per capita GDP growth ranges from a high of $5.502 \%$ in Botswana to a low of $-0.818 \%$ in Cote d'Ivoire. There are also considerable differences across countries in the degree of financial integration. For example, for the KAOPEN index when employed as an indicator for international financial openness, the highest mean index value is about $135.6 \%$ (Peru) and the lowest is $-131.9 \%$ (Colombia).

\section{B. Preliminary Analysis}

Before conducting any causality testing, it is necessary to identify the exact order of integration $\left(d_{\max }\right)$ of the variables involved in our study. To accomplish this and to provi de an analysis of sensitivity and robustness, this study performs three different standard unit root tests, namely, the Dickey and Fuller (1979) (ADF), the Phillips and Perron (1988) (PP) and the Kwiatkowski et al. (1992) (KPSS). These tests are performed on a countryby-country basis. The results are reported in Table 2 . With very few exceptions, the ADF and PP test results suggest that at the 5\% significance level, all four variables considered in this study are non-stationary in their levels but stationary in their first differences. This implies that the financial integration variables and economic growth rates are integrated of order one (I(1)). In addition, we cross-check these results applying the KPSS test, which is based on the null hypothesis of stationarity. The KPSS test results support this finding. 
To further assess the robustness of conventional unit roots tests, the Zivot and Andrews (1992) (ZA) test allowing for an endogenous structural break was also conducted. The use of this test is entirely justified by the potential of structural change in the financial integration and economic growth series over the study period, which is characterized by turbulent economic and financial crises and extreme terrorist and geopolitical events. As shown in Table 3, for the 19 developing countries, the ZA test results support the hypothesis that all variables used in the analysis are integrated of I(1) at a 5\% critical level and are thus appropriate for further analysis. In what follows, we assume all our series are unit root processes in levels and stationary in first differences.

Of note, for most of the emerging and developing countries in the sample, structural breaks around economic growth and financial integration appear to have mainly occurred at the end of the 1980s and in the mid-1990s, corresponding to the start of economic liberalization within the context of structural adjustment, initiated by international financial institutions (such as the International Monetary Fund and World Bank). Moreover, from the mid-1980s to the end of the 1990s, some of these countries experienced several crises such as the stock market crash in 1987, the Mexican currency crisis in 1994, and the Asian currency crises in July 1997.

Table 1. Descriptive statistics

\begin{tabular}{|l|l|c|c|c|c|}
\hline & & GROWTH & KAOPEN & LMF1 & LMF2 \\
\hline \multirow{4}{*}{ Argentina } & Mean & 0.966 & -0.341 & 0.870 & 0.159 \\
\cline { 2 - 6 } & Standard Deviation & 5.978 & 1.214 & 0.697 & 0.174 \\
\cline { 2 - 6 } & Maximum & 11.135 & 2.175 & 3.150 & 0.630 \\
\cline { 2 - 6 } & Minimum & -11.733 & -1.864 & 0.097 & 0.013 \\
\hline \multirow{5}{*}{ Bolivia } & Mean & 0.679 & 0.685 & 1.194 & 1.194 \\
\cline { 2 - 6 } & Standard Deviation & 2.857 & 0.837 & 0.332 & 0.332 \\
\cline { 2 - 6 } & Maximum & 5.338 & 1.383 & 1.780 & 1.780 \\
\cline { 2 - 6 } & Minimum & -6.278 & -1.864 & 0.568 & 0.568 \\
\hline \multirow{5}{*}{ Botswana } & Mean & 5.502 & 0.303 & 1.380 & 0.378 \\
\cline { 2 - 6 } & Standard Deviation & 5.278 & 1.298 & 0.335 & 0.133 \\
\cline { 2 - 6 } & Maximum & 22.253 & 2.439 & 1.868 & 0.692 \\
\cline { 2 - 6 } & Minimum & -8.691 & -1.863 & 0.549 & 0.000 \\
\hline
\end{tabular}




\begin{tabular}{|c|c|c|c|c|c|}
\hline & & GROWTH & KAOPEN & LMF1 & LMF2 \\
\hline \multirow{4}{*}{ Brazil } & Mean & 2.336 & -1.318 & 0.669 & 0.228 \\
\hline & Standard Deviation & 3.948 & 0.863 & 0.193 & 0.168 \\
\hline & Maximum & 11.311 & 0.414 & 1.018 & 0.620 \\
\hline & Minimum & -6.595 & -1.864 & 0.354 & 0.097 \\
\hline \multirow{4}{*}{ Chile } & Mean & 2.814 & -0.541 & 1.364 & 0.568 \\
\hline & Standard Deviation & 4.896 & 1.650 & 0.474 & 0.415 \\
\hline & Maximum & 10.230 & 2.439 & 2.361 & 1.546 \\
\hline & Minimum & -12.724 & -1.864 & 0.550 & 0.187 \\
\hline \multirow{4}{*}{ Colombia } & Mean & 2.105 & -1.319 & 0.583 & 0.154 \\
\hline & Standard Deviation & 2.249 & 0.752 & 0.212 & 0.131 \\
\hline & Maximum & 5.973 & 1.120 & 0.955 & 0.458 \\
\hline & Minimum & -5.830 & -1.864 & 0.312 & 0.035 \\
\hline \multirow{4}{*}{ Cote d'Ivoire } & Mean & -0.818 & -0.770 & 1.367 & 0.160 \\
\hline & Standard Deviation & 4.180 & 0.523 & 0.519 & 0.519 \\
\hline & Maximum & 7.858 & -0.113 & 2.157 & 0.357 \\
\hline & Minimum & -14.768 & -1.864 & 0.280 & 0.075 \\
\hline \multirow{4}{*}{ Costa Rica } & Mean & 2.141 & -0.061 & 1.012 & 0.138 \\
\hline & Standard Deviation & 4.180 & 1.217 & 0.425 & 0.131 \\
\hline & Maximum & 6.987 & 2.439 & 1.982 & 0.447 \\
\hline & Minimum & -9.843 & -1.864 & 0.448 & 0.035 \\
\hline \multirow{4}{*}{ Ecuador } & Mean & 1.744 & 0.2942 & 0.864 & 0.168 \\
\hline & Standard Deviation & 3.172 & 0.985 & 0.985 & 0.080 \\
\hline & Maximum & 10.806 & 2.439 & 1.444 & 0.351 \\
\hline & Minimum & -6.635 & -1.072 & 0.447 & 0.055 \\
\hline \multirow{4}{*}{ Egypt } & Mean & 3.257 & -0.378 & 1.023 & 0.181 \\
\hline & Standard Deviation & 2.757 & 1.850 & 0.392 & 0.128 \\
\hline & Maximum & 12.253 & 2.439 & 1.819 & 0.396 \\
\hline & Minimum & -1.344 & -1.864 & 0.305 & 0.004 \\
\hline \multirow{4}{*}{ South Korea } & Mean & 5.418 & -0.502 & 0.718 & 0.165 \\
\hline & Standard Deviation & 3.449 & 0.620 & 0.336 & 0.336 \\
\hline & Maximum & 10.071 & 0.942 & 1.626 & 0.666 \\
\hline & Minimum & -7.524 & -1.168 & 0.321 & 0.017 \\
\hline
\end{tabular}




\begin{tabular}{|c|c|c|c|c|c|}
\hline & & GROWTH & KAOPEN & LMF1 & LMF2 \\
\hline \multirow{4}{*}{ Malaysia } & Mean & 3.912 & 0.911 & 1.410 & 0.538 \\
\hline & Standard Deviation & 3.647 & 1.097 & 1.097 & 0.293 \\
\hline & Maximum & 9.033 & 2.439 & 2.345 & 1.172 \\
\hline & Minimum & -9.635 & -1.168 & 0.617 & 0.208 \\
\hline \multirow{4}{*}{ Mexico } & Mean & 1.526 & 0.911 & 0.646 & 0.207 \\
\hline & Standard Deviation & 3.381 & 1.307 & 0.263 & 0.179 \\
\hline & Maximum & 6.980 & 2.439 & 1.187 & 0.633 \\
\hline & Minimum & -7.587 & -1.863 & 0.229 & 0.036 \\
\hline \multirow{4}{*}{ Morocco } & Mean & 2.410 & -1.309 & 0.942 & 0.182 \\
\hline & Standard Deviation & 4.127 & 0.463 & 0.306 & 0.179 \\
\hline & Maximum & 10.537 & -0.113 & 1.339 & 0.586 \\
\hline & Minimum & -8.025 & -1.864 & 0.350 & 0.036 \\
\hline \multirow{4}{*}{ Paraguay } & Mean & 2.314 & -0.352 & 1.057 & 0.106 \\
\hline & Standard Deviation & 4.022 & 0.179 & 0.727 & 0.040 \\
\hline & Maximum & 11.126 & 1.383 & 3.013 & 0.177 \\
\hline & Minimum & -5.879 & -1.864 & 0.490 & 0.039 \\
\hline \multirow{4}{*}{ Peru } & Mean & 0.484 & 1.3567 & 0.893 & 0.193 \\
\hline & Standard Deviation & 5.423 & 0.040 & 0.202 & 0.164 \\
\hline & Maximum & 2.439 & 10.760 & 1.211 & 0.587 \\
\hline & Minumum & -1.864 & -13.870 & 0.526 & 0.033 \\
\hline \multirow{4}{*}{ Tunisia } & Mean & 3.038 & -1.068 & 1.117 & 0.500 \\
\hline & Standard Deviation & 3.440 & 0.313 & 0.324 & 0.179 \\
\hline & Maximum & 15.826 & -0.113 & 1.596 & 0.778 \\
\hline & Minimum & -4.502 & -1.169 & 0.537 & 0.201 \\
\hline \multirow{4}{*}{ Uruguay } & Mean & 2.066 & 1.015 & 1.138 & 0.108 \\
\hline & Standard Deviation & 4.696 & 1.423 & 0.583 & 0.101 \\
\hline & Maximum & 8.574 & 2.439 & 2.483 & 0.392 \\
\hline & Minimum & -10.853 & -1.864 & 0.280 & 0.020 \\
\hline \multirow{4}{*}{ Venezuela } & Mean & -0.014 & 0.207 & 1.060 & 0.180 \\
\hline & Standard Deviation & 5.501 & 1.346 & 0.386 & 0.177 \\
\hline & Maximum & 16.196 & 2.439 & 1.964 & 0.656 \\
\hline & Minimum & -10.896 & -1.864 & 0.459 & 0.0132 \\
\hline
\end{tabular}

(Note) GROWTH: per capita GDP growth, KAPOEN: capital account openness index, LMF1: aggregate stock of external assets and liabilities to GDP, LMF2: sum of total foreign equity assets and liabilities as a share of GDP. 


\begin{tabular}{|c|c|c|c|c|c|c|c|c|c|c|c|c|c|c|c|c|c|c|c|c|c|}
\hline \multirow{4}{*}{ 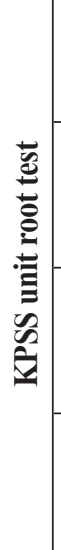 } & 空 & $\begin{array}{l}\text { శీ } \\
\text { } \\
\hat{0}\end{array}$ & $\begin{array}{l}\infty \\
\infty \\
\varnothing \\
0 \\
0\end{array}$ & $\frac{m}{0}$ & $\begin{array}{l}\infty \\
\infty \\
0 \\
0\end{array}$ & $\begin{array}{l}8 \\
0 \\
0\end{array}$ & $\begin{array}{l}\infty \\
\infty \\
\stackrel{n}{0}\end{array}$ & $\begin{array}{l}\infty \\
\stackrel{5}{0} \\
0\end{array}$ & $\begin{array}{l}\infty \\
\stackrel{0}{0} \\
0 \\
0\end{array}$ & $\begin{array}{l}\stackrel{n}{\check{n}} \\
\stackrel{0}{\circ}\end{array}$ & $\begin{array}{l}\infty \\
0 \\
0 \\
0\end{array}$ & $\begin{array}{l}\frac{0}{N} \\
0\end{array}$ & $\begin{array}{l}+ \\
+ \\
0\end{array}$ & 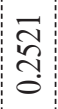 & $\begin{array}{l}2 \\
0 \\
0\end{array}$ & $\begin{array}{c}\infty \\
\stackrel{N}{\widehat{N}} \\
0\end{array}$ & $\begin{array}{l}\overline{0} \\
0\end{array}$ & $\stackrel{\widetilde{\infty}}{\tilde{\infty}}$ & $\begin{array}{l}\infty \\
0 \\
0\end{array}$ & 点 & $\begin{array}{l}\stackrel{2}{0} \\
0 \\
0\end{array}$ \\
\hline & 可 & $\stackrel{1}{\Xi}$ & $\begin{array}{l}\infty \\
\stackrel{\infty}{2} \\
0\end{array}$ & $\frac{m}{0}$ & $\begin{array}{l}\infty \\
\infty \\
2 \\
0\end{array}$ & $\begin{array}{l}\tilde{n} \\
\stackrel{n}{0}\end{array}$ & $\stackrel{\infty}{\infty}$ & $\begin{array}{l}\frac{1}{0} \\
0\end{array}$ & $\begin{array}{l}\infty \\
2 \\
0 \\
0\end{array}$ & $\begin{array}{l}\tilde{n} \\
\tilde{O} \\
0\end{array}$ & \begin{tabular}{l}
$q$ \\
\multirow{0}{*}{}
\end{tabular} & $\begin{array}{l}\hat{2} \\
0 \\
0\end{array}$ & $\begin{array}{l}\widetilde{J} \\
\delta \\
0 \\
0\end{array}$ & $\begin{array}{l}\mathscr{D} \\
\stackrel{0}{1} \\
0\end{array}$ & $\begin{array}{l}\tilde{\sigma} \\
\stackrel{\sigma}{0}\end{array}$ & $\begin{array}{l}2 \\
\mathscr{0} \\
0 \\
0\end{array}$ & $\frac{\infty}{\sigma}$ & 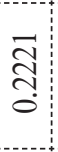 & $\begin{array}{l}\infty \\
0 \\
0 \\
0\end{array}$ & $\begin{array}{l}\infty \\
\infty \\
0\end{array}$ & $\begin{array}{l}\infty \\
0 \\
0 \\
0 \\
0\end{array}$ \\
\hline & $\begin{array}{l} \\
\\
0\end{array}$ & $\begin{array}{l}\overrightarrow{\widehat{\delta}} \\
0 \\
0\end{array}$ & ڤે & $\stackrel{\Xi}{\sigma}$ & $\begin{array}{l}\tilde{8} \\
0 \\
0\end{array}$ & $\begin{array}{l}\infty \\
\infty \\
0 \\
0\end{array}$ & $\begin{array}{c}0 \\
0 \\
0\end{array}$ & $\begin{array}{l}2 \\
\infty \\
0\end{array}$ & $\begin{array}{c}\stackrel{2}{0} \\
\dot{\sigma} \\
\dot{0}\end{array}$ & $\begin{array}{l}2 \\
\sigma \\
\sigma\end{array}$ & $\begin{array}{l}\infty \\
\infty \\
0 \\
0\end{array}$ & $\begin{array}{l}\Xi \\
\Xi \\
\end{array}$ & $\begin{array}{l}\frac{J}{\Xi} \\
0 \\
0\end{array}$ & $\stackrel{9}{\stackrel{9}{9}}$ & $\begin{array}{l}\mathscr{T} \\
\delta \\
0\end{array}$ & 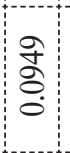 & $\begin{array}{l}\infty \\
⿱ \\
0\end{array}$ & 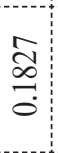 & 守 & 点 & $\begin{array}{l}\infty \\
\stackrel{\infty}{+} \\
0\end{array}$ \\
\hline & 党 & $\frac{\infty}{\sigma}$ & 乞̂ & $\begin{array}{l}\vec{\sigma} \\
\vec{\sigma}\end{array}$ & $\begin{array}{l}\infty \\
0 \\
0\end{array}$ & 仓ิ & $\begin{array}{l}\overrightarrow{\tilde{n}} \\
\stackrel{0}{0}\end{array}$ & $\frac{\mathfrak{n}}{\underset{J}{J}}$ & $\begin{array}{c}8 \\
0 \\
0\end{array}$ & $\frac{\vec{n}}{\overrightarrow{0}}$ & $\begin{array}{l}0 \\
0 \\
0\end{array}$ & $\begin{array}{l}\frac{a}{n} \\
\mathfrak{o}\end{array}$ & $\begin{array}{l}\tilde{\sigma} \\
0\end{array}$ & $\frac{7}{0}$ & $\begin{array}{l}\hat{a} \\
\dot{0}\end{array}$ & $\begin{array}{l}\bar{\sigma} \\
\tilde{n} \\
0\end{array}$ & $\begin{array}{l}\infty \\
\infty \\
0 \\
0\end{array}$ & 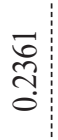 & $\stackrel{\partial}{\circ}$ & స్ & $\frac{\stackrel{\Im}{\lrcorner}}{0}$ \\
\hline \multirow{4}{*}{ 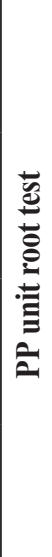 } & 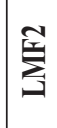 & $\begin{array}{l}\frac{a}{2} \\
\hat{2} \\
i \\
1\end{array}$ & $\begin{array}{l}\bar{\sigma} \\
\dot{\alpha} \\
\stackrel{1}{ }\end{array}$ & 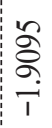 & $\begin{array}{l}\text { 足 } \\
\stackrel{5}{1}\end{array}$ & $\begin{array}{c}\mathbb{J} \\
\widetilde{d} \\
\sim\end{array}$ & $\begin{array}{l}2 \\
\infty \\
\dot{\varphi}\end{array}$ & $\begin{array}{l}\hat{D} \\
\stackrel{0}{9}\end{array}$ & $\begin{array}{c}0 \\
\stackrel{5}{0} \\
\overline{\overline{\mid}} \\
1\end{array}$ & $\begin{array}{l}\tilde{\partial} \\
\tilde{\sigma}\end{array}$ & $\begin{array}{l}\infty \\
n \\
\infty\end{array}$ & $\begin{array}{l}\hat{\partial} \\
\overrightarrow{0}\end{array}$ & 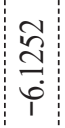 & مे & 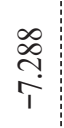 & 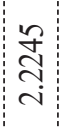 & $\begin{array}{l}D \\
D \\
b \\
\dagger\end{array}$ & \begin{tabular}{l}
$\tilde{\infty}$ \\
\hdashline
\end{tabular} & 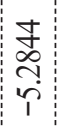 & $\frac{n}{2}$ & $\underset{f}{\tilde{f}}$ \\
\hline & $\bar{\Sigma}$ & 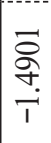 & $\begin{array}{l}\infty \\
\stackrel{\circ}{0} \\
\stackrel{0}{0}\end{array}$ & 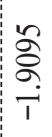 & 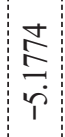 & $\begin{array}{l}\vec{n} \\
\tilde{n} \\
\hat{\imath}\end{array}$ & $\begin{array}{l}n \\
0 \\
? \\
1\end{array}$ & 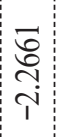 & $\begin{array}{l}\infty \\
\stackrel{8}{9} \\
\uparrow\end{array}$ & $\frac{\vec{T}}{\top}$ & $\frac{\Omega}{\rho}$ & $\begin{array}{l}\infty \\
\infty \\
\infty \\
? \\
1\end{array}$ & $\begin{array}{c}\frac{a}{2} \\
\stackrel{0}{6}\end{array}$ & $\frac{F}{\stackrel{9}{i}}$ & $\begin{array}{c}\infty \\
0 \\
\infty \\
1\end{array}$ & $\begin{array}{c}\infty \\
\stackrel{0}{\infty} \\
T\end{array}$ & 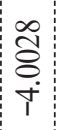 & 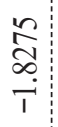 & 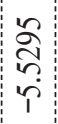 & $\begin{array}{l}\infty \\
\stackrel{\infty}{\sim} \\
\stackrel{\top}{\top}\end{array}$ & $\begin{array}{l}\stackrel{D}{ } \\
\stackrel{T}{+}\end{array}$ \\
\hline & \begin{tabular}{|l|l} 
\\
\\
0
\end{tabular} & 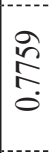 & $\frac{\beth}{\stackrel{N}{a}}$ & $\stackrel{2}{0}$ & $\begin{array}{l}\vec{a} \\
\infty \\
\infty\end{array}$ & $\begin{array}{l}\frac{n}{6} \\
0\end{array}$ & 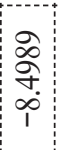 & $\begin{array}{l}+ \\
\stackrel{2}{-}\end{array}$ & 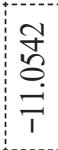 & $\begin{array}{l}\stackrel{0}{o} \\
\stackrel{+}{i} \\
2\end{array}$ & $\begin{array}{l}\vec{\sigma} \\
\text { } \\
\infty \\
\infty\end{array}$ & $\begin{array}{l}2 \\
\mathscr{J} \\
\dot{c}\end{array}$ & \begin{tabular}{l}
\multirow{2}{0}{} \\
$\stackrel{1}{9}$ \\
9
\end{tabular} & $\frac{n}{n}$ & 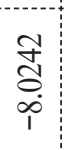 & 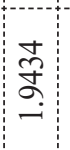 & $\underset{5}{\bar{\sigma}}$ & $\begin{array}{l}\infty \\
0 \\
0 \\
ن \\
ن\end{array}$ & $\begin{array}{l}\stackrel{n}{0} \\
\stackrel{1}{1} \\
\stackrel{1}{1}\end{array}$ & $\begin{array}{l}\vec{f} \\
\stackrel{9}{9}\end{array}$ & $\begin{array}{l}0 \\
8 \\
0 \\
i\end{array}$ \\
\hline & 公 & $\begin{array}{l}\vec{\alpha} \\
\stackrel{\alpha}{i} \\
\hat{i}\end{array}$ & $\begin{array}{l}0 \\
\infty \\
\infty \\
\infty \\
i\end{array}$ & $\stackrel{g}{i}$ & $\begin{array}{l}2 \\
0 \\
n \\
1\end{array}$ & $\begin{array}{l}\infty \\
\stackrel{\infty}{\varphi}\end{array}$ & $\frac{0}{2}$ & $\begin{array}{l}0 \\
\cdots \\
0 \\
0\end{array}$ & $\begin{array}{c}2 \\
\infty \\
0 \\
i\end{array}$ & 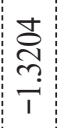 & $\stackrel{\vec{\jmath}}{\stackrel{T}{\top}}$ & $\begin{array}{c}\vec{\sigma} \\
\sigma \\
\end{array}$ & $\begin{array}{c}m \\
\alpha \\
\infty \\
\infty\end{array}$ & $\begin{array}{c}\stackrel{9}{d} \\
\infty \\
\uparrow\end{array}$ & $\begin{array}{c}\infty \\
\vec{a} \\
1\end{array}$ & $\begin{array}{c}\bar{s} \\
\stackrel{\circ}{\varphi}\end{array}$ & $\frac{\infty}{\widehat{N}}$ & مे & $\frac{n}{\infty}$ & $\begin{array}{l}\infty \\
\infty \\
\infty \\
i\end{array}$ & 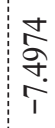 \\
\hline \multirow{6}{*}{ 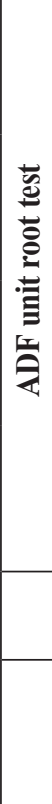 } & $\sum^{\underline{S}}$ & 京 & $\begin{array}{l}\hat{\alpha} \\
\stackrel{0}{0} \\
\hat{1}\end{array}$ & \begin{tabular}{l}
0 \\
\multirow{7}{*}{} \\
$i$
\end{tabular} & $\begin{array}{l}\tilde{n} \\
\tilde{o} \\
\uparrow\end{array}$ & 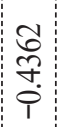 & $\begin{array}{l}\stackrel{P}{+} \\
\stackrel{p}{p}\end{array}$ & $\begin{array}{l}g \\
\stackrel{n}{n} \\
m\end{array}$ & $\begin{array}{l}2 \\
2 \\
0 \\
i\end{array}$ & 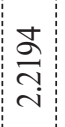 & $\begin{array}{l}\overrightarrow{8} \\
\vec{p}\end{array}$ & $\begin{array}{l}\stackrel{N}{\sigma} \\
\stackrel{\sim}{\sim}\end{array}$ & $\begin{array}{l}2 \\
\infty \\
\uparrow \\
\uparrow\end{array}$ & $\stackrel{\infty}{\stackrel{n}{n}}$ & $\frac{\infty}{\infty}$ & $\begin{array}{c}\infty \\
\mathbb{1} \\
0 \\
-\end{array}$ & 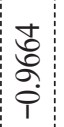 & $\begin{array}{l}0 \\
0 \\
0\end{array}$ & $\frac{\infty}{\rho}$ & 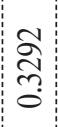 & 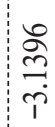 \\
\hline & $\sum^{\bar{S}}$ & స్తి & $\frac{\hat{\sigma}}{\stackrel{\sigma}{\dagger}}$ & \begin{tabular}{l}
0 \\
\multirow{\sigma}{*}{} \\
$\stackrel{1}{\varphi}$
\end{tabular} & $\begin{array}{l}\tilde{n} \\
\tilde{n} \\
\uparrow\end{array}$ & $\vec{i}$ & $\begin{array}{c}\infty \\
b \\
0\end{array}$ & $\begin{array}{l}\infty \\
\stackrel{\circ}{+}\end{array}$ & $\begin{array}{c}\hat{a} \\
\infty \\
i \\
\Gamma\end{array}$ & $\begin{array}{l}\mathfrak{2} \\
\hat{o} \\
0\end{array}$ & $\frac{\stackrel{D}{~}}{\stackrel{\Gamma}{r}}$ & $\begin{array}{l}\stackrel{0}{2} \\
\grave{\sigma}\end{array}$ & $\begin{array}{c}n \\
\delta \\
n \\
\tilde{1} \\
\tilde{1}\end{array}$ & $\frac{\infty}{\stackrel{1}{\sigma}}$ & $\begin{array}{c}\tilde{b} \\
\dot{1}\end{array}$ & $\begin{array}{c}\hat{\sigma} \\
\hat{\jmath} \\
\hat{\varphi}\end{array}$ & $\begin{array}{l}2 \\
2 \\
\stackrel{2}{\uparrow}\end{array}$ & 号 & $\frac{\infty}{\stackrel{े}{+}}$ & $\begin{array}{l}+ \\
0 \\
0 \\
0\end{array}$ & $\begin{array}{c}d \\
\mathbb{d} \\
i \\
i\end{array}$ \\
\hline & 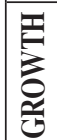 & 昌 & 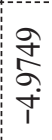 & 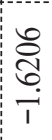 & $\begin{array}{l}0 \\
\stackrel{0}{m} \\
\stackrel{f}{*}\end{array}$ & $\frac{\widehat{q}}{\stackrel{f}{T}}$ & $\begin{array}{l}\infty \\
\stackrel{2}{n} \\
i \\
r\end{array}$ & 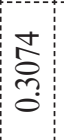 & 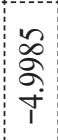 & 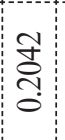 & $\begin{array}{l}\stackrel{\Upsilon}{+} \\
\stackrel{1}{*}\end{array}$ & $\frac{\overrightarrow{2}}{2}$ & $\begin{array}{l}\frac{n}{\widetilde{f}} \\
\stackrel{f}{f}\end{array}$ & $\begin{array}{l}n \\
\stackrel{n}{o}\end{array}$ & $\begin{array}{l}\hat{0} \\
2 \\
\hat{1}\end{array}$ & $\begin{array}{l}\infty \\
\stackrel{\infty}{n} \\
\stackrel{0}{0}\end{array}$ & $\begin{array}{l}\vec{F} \\
D \\
+\end{array}$ & $\begin{array}{l}\vec{D} \\
\frac{\nabla}{T}\end{array}$ & \begin{tabular}{l}
$\vec{b}$ \\
\multirow{n}{r}{}
\end{tabular} & $\frac{\tilde{\sigma}}{+}$ & 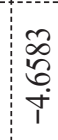 \\
\hline & 亘 & 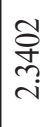 & 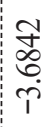 & $\begin{array}{l}8 \\
8 \\
\infty \\
i\end{array}$ & $\begin{array}{l}\mathcal{N} \\
\tilde{n} \\
\uparrow\end{array}$ & $\begin{array}{l}\frac{a}{m} \\
\frac{m}{9}\end{array}$ & $\begin{array}{l}\infty \\
\infty \\
\infty \\
i\end{array}$ & $\stackrel{2}{\curvearrowright}$ & $\begin{array}{l}a \\
i \overrightarrow{2} \\
i \\
i\end{array}$ & 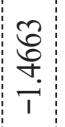 & $\frac{m}{m}$ & \begin{tabular}{l}
$\stackrel{\infty}{+}$ \\
\hdashline
\end{tabular} & $\begin{array}{c}\infty \\
\stackrel{T}{†}\end{array}$ & $\begin{array}{c}\infty \\
\widehat{a} \\
\hat{1}\end{array}$ & $\begin{array}{l}\sigma \\
\sigma \\
+\end{array}$ & $\begin{array}{l}\infty \\
0 \\
0 \\
1\end{array}$ & 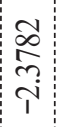 & $\frac{2}{2}$ & $\begin{array}{l}\infty \\
\stackrel{\infty}{i} \\
\stackrel{1}{i}\end{array}$ & $\begin{array}{l}\tilde{n} \\
\alpha \\
i\end{array}$ & 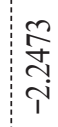 \\
\hline & & ब्व & $\triangleleft$ & $\begin{array}{l}\overrightarrow{0} \\
\overrightarrow{0}\end{array}$ & $\triangleleft$ & $\vec{D}$ & $\triangleleft$ & $\mid \begin{array}{l}\overrightarrow{0} \\
\bar{z}\end{array}$ & $\triangleleft$ & $\left|\begin{array}{l}\overrightarrow{0} \\
\vec{d}\end{array}\right|$ & $\triangleleft$ & $\begin{array}{l}\overline{0} \\
\vec{z}\end{array}$ & $\triangleleft$ & \begin{tabular}{|l|}
$\overrightarrow{0}$ \\
$\vec{z}$
\end{tabular} & $\triangleleft$ & 曾 & $\triangleleft$ & $\begin{array}{l}\vec{D} \\
\stackrel{\Xi}{\Omega}\end{array}$ & $\triangleleft$ & \begin{tabular}{|l|}
$\overrightarrow{0}$ \\
$\vec{z}$
\end{tabular} & $\triangleleft$ \\
\hline & 己 & & E & 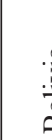 & ڤ్ & है & 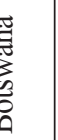 & 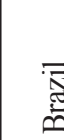 & ที & & & छี & छิ & ठั & $\begin{array}{l}\stackrel{0}{0} \\
\stackrel{0}{0}\end{array}$ & 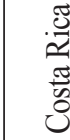 & 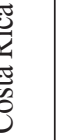 & II & & & 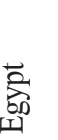 \\
\hline
\end{tabular}




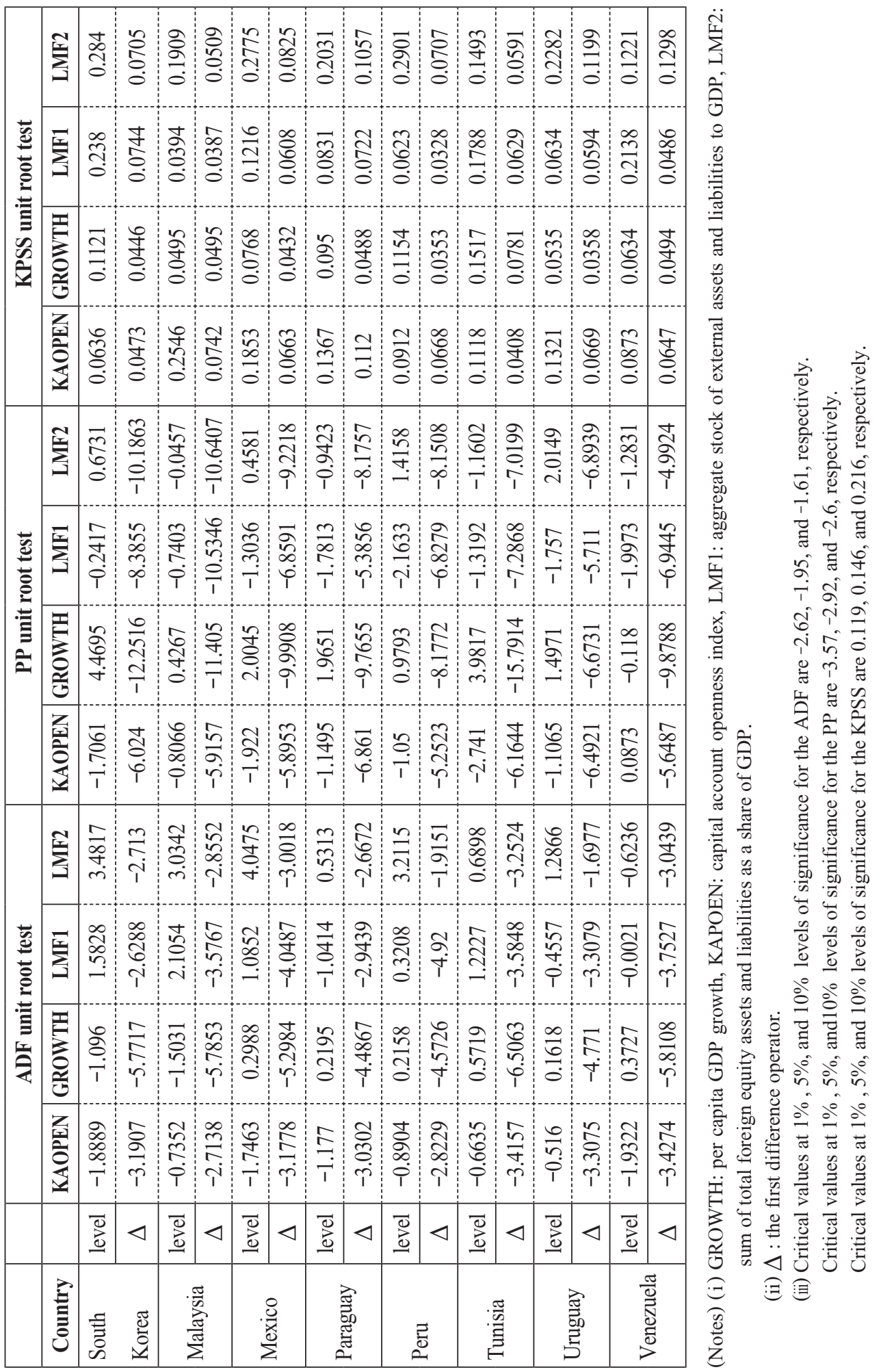


Table 3. Results of Zivot unit root test

\begin{tabular}{|c|c|c|c|c|c|c|c|c|c|}
\hline \multirow{2}{*}{ Country } & & \multicolumn{2}{|c|}{ KAOPEN } & \multicolumn{2}{|c|}{ LMF1 } & \multicolumn{2}{|c|}{ LMF2 } & \multicolumn{2}{|c|}{ GROWTH } \\
\hline & & Statistics & Break & atistics & Break & Statistics & Break & tatistics & Break \\
\hline \multirow{2}{*}{ Argentina } & evel & -3.723 & 1992 & 8.4243 & 2001 & -3.192 & 2000 & -4.0539 & 1990 \\
\hline & $\Delta$ & 4.2801 & 1996 & 5.3532 & 2003 & 5.9512 & 2003 & 5.9262 & 001 \\
\hline \multirow{2}{*}{ Bolivia } & level & 3.3778 & 1980 & -2.755 & 1980 & -2.755 & 980 & -3.2814 & 977 \\
\hline & $\Delta$ & 4.6456 & 1984 & 3.0446 & 1991 & -3.0446 & 991 & -5.7402 & 982 \\
\hline \multirow{2}{*}{ Botswan } & level & 3.6055 & 1995 & -1.9596 & 1980 & -3.6792 & 1983 & -6.0189 & 89 \\
\hline & $\Delta$ & 3.9102 & 1993 & 5.3009 & 2005 & -5.1085 & 1976 & -5.9379 & 986 \\
\hline \multirow{2}{*}{ Brazil } & level & 5.2021 & 2001 & 3.5684 & 1986 & -4.1414 & 1998 & -4.8374 & 980 \\
\hline & $\Delta$ & -4.3617 & 2008 & -4.5621 & 1983 & -4.6446 & 1996 & -5.6885 & 982 \\
\hline \multirow{2}{*}{ Chile } & level & -4.8279 & 2000 & .5636 & 1990 & -2.2268 & 1997 & -3.974 & 997 \\
\hline & $\Delta$ & 3.6491 & 1997 & 4.3587 & 1985 & -4.8935 & 2002 & -4.8724 & 974 \\
\hline \multirow{2}{*}{ Colombia } & level & -5.3883 & 2003 & 3.6547 & 1996 & -2.5766 & 2002 & -4.024 & 2002 \\
\hline & $\Delta$ & -6.1607 & 2007 & -4.1478 & 2002 & -5.8973 & 1995 & -4.7681 & 998 \\
\hline \multirow{2}{*}{ Cote d'Ivoire } & level & : & 1900 & & 1700 & & 1997 & & 皮 \\
\hline & $\Delta$ & -4.7655 & 1976 & 4.8462 & 1993 & -3.1675 & 2003 & 485 & 981 \\
\hline \multirow{2}{*}{ Costa Rica } & level & -3.0203 & 1993 & -3.3526 & 1980 & -2.4505 & 1997 & -4.1886 & 1979 \\
\hline & $\Delta$ & -3.8378 & 1008 & -4.2508 & 1081 & -4.1334 & 1995 & -5.8947 & 981 \\
\hline \multirow{2}{*}{ Ecuado } & lever & -3.3814 & 2003 & .4973 & 1900 & .8902 & 1974 & 02 & 976 \\
\hline & $\Delta$ & -3.2314 & 2009 & -3.852 & 1989 & -7.0721 & 1999 & -5.6979 & 1974 \\
\hline \multirow{2}{*}{ Egypt } & level & -4.8225 & 1993 & -3.7137 & 1978 & -4.1183 & 1990 & -4.5246 & 985 \\
\hline & $\Delta$ & -3.5922 & 2001 & -4.096 & 1 & -3.6788 & 2007 & 87 & 976 \\
\hline \multirow{2}{*}{ South Korea } & level & -3.8175 & 1995 & 2.7043 & 1985 & -1.5829 & 1997 & & 982 \\
\hline & $\Delta$ & -3.6441 & 1998 & -3.8484 & 1991 & -6.6571 & 1996 & -5.8285 & 1997 \\
\hline \multirow{2}{*}{ Malaysia } & level & -3.2434 & 1981 & -4.2128 & 1988 & -3.0483 & 1992 & -3.9161 & 988 \\
\hline & $\Delta$ & -4.385 & 1999 & -4.7127 & 1986 & -5.1785 & 1995 & -5.9896 & 986 \\
\hline \multirow{2}{*}{ Mexico } & level & -6.1971 & 1981 & -2.9894 & 1981 & -2.4881 & 2003 & -5.0386 & 1981 \\
\hline & $\Delta$ & -4.3622 & 1985 & -5.8946 & 1986 & -7.2094 & 2006 & -5.4049 & 1985 \\
\hline \multirow{2}{*}{ Morocco } & level & -4.9404 & 1985 & -3.4371 & 1980 & -3.5686 & 2000 & -3.8615 & 1978 \\
\hline & $\Delta$ & -7.911 & 1994 & -4.5083 & 1984 & -5.3271 & 2008 & -6.4005 & 1976 \\
\hline \multirow{2}{*}{ Paraguay } & level & -4.1781 & 1996 & -4.9518 & 1999 & -3.2572 & 1993 & -4.3716 & 198 \\
\hline & $\Delta$ & -3.9388 & 1987 & -4.0956 & 2002 & -4.6054 & 1999 & -4.8576 & 1979 \\
\hline \multirow{2}{*}{ Peru } & level & -4.2097 & 1991 & -5.9468 & 1976 & -1.6128 & 1980 & -4.3161 & 1981 \\
\hline & $\Delta$ & -3.9692 & 1989 & -5.2349 & 1988 & -5.5881 & 1992 & -4.7741 & 1989 \\
\hline
\end{tabular}




\begin{tabular}{|l|c|c|c|c|c|c|c|c|c|}
\hline \multirow{2}{*}{ Country } & & \multicolumn{2}{|c|}{ KAOPEN } & \multicolumn{2}{c|}{ LMF1 } & \multicolumn{2}{c|}{ LMF2 } & \multicolumn{2}{c|}{ GROWTH } \\
\cline { 2 - 9 } & & Statistics & Break & Statistics & Break & Statistics & Break & Statistics & Break \\
\hline \multirow{3}{*}{ Tunisia } & level & -4.1144 & 1991 & -3.7604 & 1981 & -4.5997 & 1980 & -5.4073 & 1995 \\
\cline { 2 - 10 } & $\Delta$ & -5.0583 & 1994 & -4.8882 & 1986 & -4.29 & 1984 & -7.1095 & 2007 \\
\hline \multirow{3}{*}{ Uruguay } & level & -3.6196 & 1979 & -3.1957 & 2009 & -3.1446 & 2005 & -4.4913 & 1980 \\
\cline { 2 - 10 } & $\Delta$ & -4.9641 & 1994 & -3.9925 & 2003 & -3.5467 & 1999 & -5.0758 & 2001 \\
\hline \multirow{3}{*}{ Venezuela } & level & -3.954 & 1995 & -3.1714 & 2003 & -2.866 & 1995 & -4.663 & 2003 \\
\cline { 2 - 9 } & $\Delta$ & -4.302 & 2000 & -4.2146 & 2003 & -6.3845 & 2002 & -6.34 & 2006 \\
\hline
\end{tabular}

(Notes) (i) GROWTH: per capita GDP growth, KAPOEN: capital account openness index, LMF1: aggregate stock of external assets and liabilities to GDP, LMF2: sum of total foreign equity assets and liabilities as a share of GDP.

(ii) $\Delta$ : the first difference operator.

(iii) Critical values at $1 \%, 5 \%$, and $10 \%$ levels of significance for the ZA are $-5.34,-4.8$, and -4.58 , respectively.

\section{Results}

\section{A. Linear causality test results}

Having established the integration properties of each of the variables under consideration, we apply the causality approach developed by Toda and Yamamoto (1995). However, it is also well-known that this testing method is very sensitive to the number of lags included in the regression. Thus, prior to causality analysis, we have to determine the appropriate lag length for the various models. To that end, we employed four lag selection information criteria often employed in the literature, namely, the Aikaike Information Criterion (AIC), Schwarz Information Criterion (SIC), Final Prediction Error (FPE) and Hannan Quinn (HQ) information criterion. To conserve space, these results are not reported here but are available from the authors upon request.

Table 4 reports results of the Granger non-causality test from the Toda and Yamamoto (1995) procedure. The MWALD test statistics regarding the causal relation between financial integration and growth in the 19 emerging and developing countries (rows) that conform to our sample and their corresponding significance levels are presented in the first three columns of results. Of the 19 countries, the results show that none of 
the financial integration indicators causes economic growth in the cases of Argentina, Botswana, Brazil, Chile, Cote d'Ivoire, Colombia, Egypt, South Korea, Morocco, and Paraguay. Similar findings for Botswana are reported by Ahmed and Mmolainyane (2014). Further, the same picture is observed for Bolivia, Costa Rica, Ecuador, Malaysia, Tunisia, Uruguay, and Venezuela, in which only one of the financial openness proxies causes economic growth. For Mexico and Peru, on the other hand, we found greater evidence against the null hypothesis of an absence of Granger causality from financial integration to growth. In fact, in each of these two countries, Granger causality was detected in two of the three proxies of financial integration.

The three columns on the right in Table 4 report the results regarding the presence of a causal link from growth to financial integration. The significance of the $\rho$-values for the MWALD statistic provides evidence against the null hypothesis of no causality running from the capital account openness index to real GDP growth in Bolivia, Cote d'Ivoire, Colombia, and Ecuador. This evidence is even stronger-in terms of number of countries and significance levels - for the aggregate stock of external assets and liabilities as a percentage of GDP. Moreover, the evidence favorable to a causal link from the sum of total foreign equity assets and liabilities as a share of GDP to growth is mainly found in developing countries (Bolivia, Chile, South Korea, and Paraguay). It can also be observed that the null hypothesis of the lack of causality from financial integration to growth cannot be rejected in Bolivia for any of the financial integration indicators analyzed.

Taken together, the results displayed in Table 4 reveal the following findings. Based on the capital account openness index as proxy for financial integration, we found evidence of linear Granger causality for six countries, namely, Bolivia, Cote d'Ivoire, Colombia, Ecuador, Mexico, and Uruguay. In Cote d'Ivoire, Colombia, and Ecuador, causality runs from economic growth to financial integration; in Mexico and Uruguay, causality runs from financial integration to economic growth, and in Bolivia, we found that financial integration and economic growth are mutually causal. While using the aggregate stock of external assets and liabilities (as a percentage of GDP) as a measure of financial integration, the results indicate that there is a unidirectional causality running from financial integration to economic growth in Costa Rica, Mexico, Peru, and Venezuela. However, in the case of Bolivia, Cote d'Ivoire, South Korea, Malaysia, and Uruguay, causality runs from economic growth to financial integration. Turning now to the sum of total foreign equity assets and liabilities as a share of GDP as a proxy for financial integration, the results show evidence of unidirectional causality running 
from financial integration to economic growth in Ecuador, Malaysia, Peru, and Tunisia, whereas unidirectional causality runs from economic growth to financial integration in Bolivia, Chile, South Korea, and Paraguay. Therefore, our results highlight that the causality link between financial integration and economic growth is sensitive to the indicator of financial integration chosen.

In sum, it can be stated that except for Mexico and Peru, the results from the linear Granger causality tests do not provide strong evidence supporting the view that financial integration is an important determinant of economic growth in developing countries. These results differ from those in the studies by Rajan and Zingales (1998), Prasad et al. (2003), Bonfiglioli (2008), Baltagi et al. (2009), Gehringer (2012), and Sandri (2014), which suggested that there was a significant correlation between financial integration and economic growth variables. However, our results are quite consistent with the studies of Edison et al. (2002), Alfaro et al. (2005), Bussière and Fratzscher (2008), and Gourinchas and Jeanne (2013).

This may imply the failure of prior linear tests in capturing the relationship between financial integration and economic growth. Therefore, as stressed earlier, we also apply a nonlinear approach in this study to further examine the issue.

Table 4. Granger (non-)causality MWALD test statistics

\begin{tabular}{|c|c|c|c|c|c|c|}
\hline \multirow[t]{2}{*}{ Country } & \multicolumn{3}{|c|}{$\begin{array}{c}\text { Financial integration } \rightarrow \\
\text { Economic growth }\end{array}$} & \multicolumn{3}{|c|}{$\begin{array}{l}\text { Economic growth } \longrightarrow \\
\text { Financial integration }\end{array}$} \\
\hline & KAOPEN & LMF1 & LMF2 & KAOPEN & LMF1 & LMF2 \\
\hline Argentina & 2.2 & 3.0 & 0.9 & 2.0 & 3.8 & 0.6 \\
\hline Bolivia & $6.4 * *$ & 0.74 & 0.74 & $7.0^{* *}$ & $11.2 * * *$ & $11.2 * * *$ \\
\hline Botswana & 1.9 & 1.8 & 4.1 & 0.34 & 1.5 & 0.88 \\
\hline Brazil & 3.3 & 1.8 & 5.7 & 1.0 & 1.5 & 1.7 \\
\hline Chile & 1.3 & 2.4 & 1.2 & 3.1 & 3.6 & $6.3 * *$ \\
\hline Cote d'Ivoire & 0.21 & 0.84 & 0.043 & $8.5 * *$ & $7.2 * *$ & 1.1 \\
\hline Colombia & 2.0 & 2.5 & 0.043 & $5.9^{*}$ & 2.0 & 1.1 \\
\hline Costa Rica & 2.0 & $7.5 * *$ & 0.043 & 2.3 & 0.41 & 1.1 \\
\hline Ecuador & 1.7 & 2.3 & $10.8 * * *$ & $5.2^{*}$ & 1.1 & 2.6 \\
\hline Egypt & 0.64 & 2.3 & 0.45 & 0.15 & 1.1 & 1.7 \\
\hline South Korea & 0.66 & 3.4 & 2.9 & 0.65 & $13.4^{* * *}$ & $6.7 * *$ \\
\hline Malaysia & 1.6 & 4.4 & $11.0 * *$ & 0.61 & $9.8 * * *$ & 3.9 \\
\hline
\end{tabular}




\begin{tabular}{|l|c|c|c|c|c|c|}
\hline \multirow{2}{*}{ Country } & \multicolumn{3}{|c|}{$\begin{array}{c}\text { Financial integration } \rightarrow \\
\text { Economic growth }\end{array}$} & \multicolumn{3}{c|}{$\begin{array}{c}\text { Economic growth } \rightarrow \\
\text { Financial integration }\end{array}$} \\
\hline & KAOPEN & LMF1 & LMF2 & KAOPEN & LMF1 & LMF2 \\
\hline Mexico & $9.4^{* * *}$ & $7.4^{* *}$ & 4.2 & 0.52 & 2.3 & 3.4 \\
\hline Morocco & 1.1 & 3.3 & 3.8 & 1.3 & 3.9 & 1.8 \\
\hline Paraguay & 0.12 & 1.5 & 3.9 & 1.6 & 3.7 & $4.7 *$ \\
\hline Peru & 2.6 & $12.4 * * *$ & $6.4 * *$ & 2.1 & 4.2 & 0.81 \\
\hline Tunisia & 0.65 & 3.4 & $9.2^{* * *}$ & 0.33 & 2.4 & 0.99 \\
\hline Uruguay & $8.3 * *$ & 2.9 & 4.1 & 0.71 & $27.0 * * *$ & 3.0 \\
\hdashline Venezuela & 3.3 & $6.4 * *$ & 0.7 & 1.7 & 2.1 & 0.73 \\
\hline
\end{tabular}

(Notes) (i) GROWTH: per capita GDP growth, KAPOEN: capital account openness index, LMF1: aggregate stock of external assets and liabilities to GDP, LMF2: sum of total foreign equity assets and liabilities as a share of GDP.

(ii) For $\mathrm{X} \rightarrow \mathrm{Y}, \mathrm{H} 0$ : $\mathrm{X}$ does not cause $\mathrm{Y}$.

(iii) $* * *, * *$ and $*$ denote rejection of the null hypothesis at the $1 \%, 5 \%$, and $10 \%$ significance levels, respectively.

\section{B. Nonlinear Granger causality test results}

Before implementing the nonlinear Granger causality test recently developed by Kyrtsou and Labys (2006), it is crucial to first determine whether the data are characterized by nonlinearities. For this purpose, the BDS nonlinearity test proposed by Brock et al. (1987) was performed on the residual series of VAR models to assess the validity of the iid assumption. The results reported in Table 5 reveal that irrespective of the implemented dimension, the null hypothesis of linearity should be rejected at the $1 \%$ level of significance for all series under consideration. Such a result signifies that instead of the standard Granger causality test, the nonlinear Granger causality test would appear to be more appropriate.

Applying the Kyrtsou and Labys (2006) nonlinear Granger causality test yields the results reported in Table 6 . The results indicate that none of the financial integration proxy variables causes economic growth in the cases of Brazil, Chile, Cote d'Ivoire, Costa Rica, Egypt, Ecuador, South Korea, Malaysia, Mexico, Paraguay, and Peru. As for Argentina, Bolivia, Colombia, Tunisia, Morocco, and Venezuela, however, we found strong empirical support for a nonlinear causal link from financial integration to growth. In fact, for each of these cases listed above, Granger causality was detected in 
at least two of the three financial integration indicators. Nevertheless, in these countries, the results do not show a uniform structure. For instance, while the aggregate stock of external assets and liabilities as well as the stock of liabilities cause economic growth in Colombia, Tunisia, and Morocco, either the capital account openness index or the stock of external assets and liabilities cause economic growth in Argentina and Venezuela.

Compared with the linear Granger causality test result, the nonlinear test result for Bolivia is consistent. For Argentina, Bolivia, Colombia, Tunisia, Morocco, and Venezuela, the results are in sharp contrast to those obtained by the Toda-Yamamoto test, which show no causal relationship running from financial integration indicators to economic growth. These results confirm that the causal relation between financial integration and economic growth is not strictly linear but also nonlinear. However, it is worth noting that such findings deserve further substantive investigations which could help support or refute the results presented here.

Regarding causality from economic growth to financial integration, the results clearly indicate that at the $10 \%$ significance level, financial integration is not sensitive to economic growth in Colombia, Morocco, Tunisia, Venezuela, and Peru, in which none of the financial integration indicators is associated with the percapita GDP growth. For the remaining 13 countries, however, the results provide evidence of a causal link from economic growth to financial integration. Most notably, this evidence is stronger in Cote d'Ivoire and Malaysia, in which all the financial integration indicators are associated with percapita GDP growth. Furthermore, albeit by only one indicator, a two-way Granger causality between financial integration and economic growth was observed in Bolivia and Uruguay.

In summary, according to the nonlinear Granger causality tests, there seems to be evidence, albeit relatively weak, supporting the view that financial integration is an important determinant of economic growth in developing countries. That is, more extensive financial integration will lead to more economic growth. These findings are complementary to those of Kose et al. (2011) and Chen and Quang (2014), who showed that statistically significant relations between financial integration and economic growth exist when allowance is made for nonlinearities. Thus, the results presented here reinforce related literature by showing that financial integration and economic growth interact in a nonlinear fashion.

Based on our results, it seems promising for future research to investigate the specific type of nonlinearities that characterize the relationship between financial integration and economic growth. It would also be interesting for future research to examine the impact 
of nonlinearity on the performance of the linear modeling techniques that have been employed so far in the related literature. This exercise could provide an explanation for the inconclusive results reported by previous research (Eichengreen 2001, Prasad et al. 2003, Kose et al. 2010, Schularick and Steger 2010, Bumann et al. 2013). 


\begin{tabular}{|c|c|c|c|c|c|c|c|c|c|c|c|c|c|c|c|c|c|c|c|c|c|}
\hline$\sim$ & 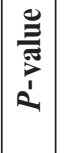 & 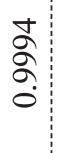 & o & $\begin{array}{l}\infty \\
\infty \\
0\end{array}$ & సे & $\begin{array}{c}0 \\
d \\
\sim \\
\sim\end{array}$ & $\begin{array}{c}0 \\
d \\
\\
\text { d }\end{array}$ & $\begin{array}{c}0 \\
d \\
d \\
d\end{array}$ & $\begin{array}{c:}0 \\
\\
\stackrel{d}{d} \\
\end{array}$ & $\begin{array}{l}o \\
d \\
\stackrel{d}{c}\end{array}$ & $\begin{array}{c}o \\
\stackrel{b}{d} \\
\stackrel{d}{d}\end{array}$ & 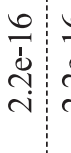 & 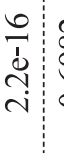 & 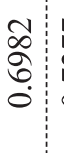 & $\frac{\hat{N}}{\hat{n}}$ & 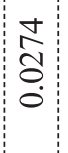 & 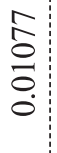 & $\begin{array}{l}0 \\
d \\
\sim \\
\sim\end{array}$ & $\begin{array}{l}o \\
d \\
\mathrm{~d} \\
\mathrm{v}\end{array}$ & $\begin{array}{c:c}0 \\
\\
\\
\end{array}$ & 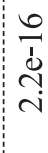 \\
\hline 主 & 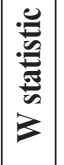 & $\begin{array}{l:} \\
0 \\
1 \\
0 \\
0 \\
\infty\end{array}$ & $\begin{array}{l}\overline{8} \\
0\end{array}$ & $\begin{array}{c}\infty \\
0 \\
0\end{array}$ & 峁 & $\begin{array}{l}\tilde{\rho} \\
\infty \\
\dot{n}\end{array}$ & $\begin{array}{c}\stackrel{m}{\infty} \\
\dot{m}\end{array}$ & 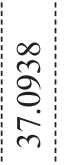 & $\begin{array}{l}\stackrel{n}{n} \\
\stackrel{p}{+}\end{array}$ & $\begin{array}{l}\vec{n} \\
\stackrel{n}{n}\end{array}$ & 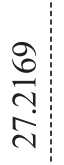 & 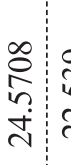 & $\begin{array}{c:c} & 0 \\
n & 0 \\
& 0\end{array}$ & 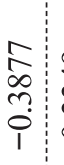 & mે? & $\begin{array}{c}n \\
\stackrel{n}{N} \\
i \\
i\end{array}$ & 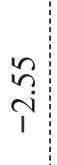 & $\begin{array}{l}\infty \\
b \\
\stackrel{0}{2}\end{array}$ & $\begin{array}{l}0 \\
0 \\
\pm \\
\pm\end{array}$ & $\begin{array}{l}\text { Dे } \\
\text { సે } \\
\\
\end{array}$ & $\begin{array}{l}\infty \\
\stackrel{\infty}{N} \\
\stackrel{-}{0}\end{array}$ \\
\hline & 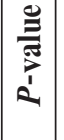 & $\begin{array}{c:}0 \\
\\
\\
\end{array}$ & $\begin{array}{c}0 \\
\mathfrak{d} \\
\sim \\
\sim\end{array}$ & $\begin{array}{c}0 \\
d \\
d \\
\sim\end{array}$ & $\begin{array}{c}0 \\
0 \\
d \\
\sim\end{array}$ & $\begin{array}{c}0 \\
j \\
\sim \\
c\end{array}$ & $\begin{array}{c}0 \\
d \\
d \\
\end{array}$ & $\begin{array}{c}0 \\
d \\
d \\
\end{array}$ & 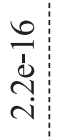 & $\begin{array}{l}\sigma \\
d \\
\sim \\
\sim\end{array}$ & $\begin{array}{c:}o \\
\end{array}$ & 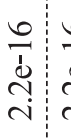 & 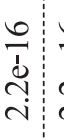 & 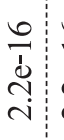 & $\begin{array}{c:}o \\
\end{array}$ & 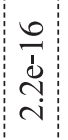 & $\begin{array}{c:c}0 \\
\\
\stackrel{1}{d} \\
\end{array}$ & $\begin{array}{c}0 \\
\dot{d} \\
\stackrel{\sim}{N}\end{array}$ & $\begin{array}{l}o \\
d \\
\sim \\
\sim\end{array}$ & $\begin{array}{c:c}0 & \\
& \\
& \\
& \end{array}$ & 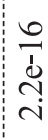 \\
\hline $1=$ & 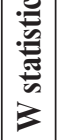 & 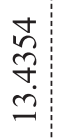 & $\begin{array}{l}\mathbb{N} \\
\frac{N}{n} \\
2\end{array}$ & $\begin{array}{c}\stackrel{2}{n} \\
\stackrel{n}{\beth}\end{array}$ & $\begin{array}{l} \pm \\
\vec{\sim} \\
\stackrel{\sim}{\sim}\end{array}$ & $\begin{array}{l}\tilde{\rho} \\
\infty \\
\stackrel{\rho}{n}\end{array}$ & $\begin{array}{l}\stackrel{+}{\infty} \\
\stackrel{+}{m}\end{array}$ & 交 & $\begin{array}{l}n \\
\stackrel{n}{n} \\
\stackrel{o}{+}\end{array}$ & $\begin{array}{l}\infty \\
\stackrel{2}{0} \\
\stackrel{2}{c}\end{array}$ & $\begin{array}{l}\vec{\beth} \\
\stackrel{\alpha}{\infty}\end{array}$ & 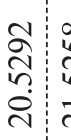 & $\begin{array}{c:c}\infty \\
n \\
n \\
\end{array}$ & $\begin{array}{l}\tilde{2} \\
\ddot{2}\end{array}$ & $\begin{array}{l}n \\
\tilde{n} \\
\end{array}$ & 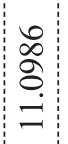 & \begin{tabular}{l}
$\stackrel{2}{\hat{n}}$ \\
\multirow{2}{0}{} \\
$\stackrel{0}{n}$
\end{tabular} & $\begin{array}{l}\infty \\
\stackrel{D}{\infty} \\
\infty \\
\infty \\
n\end{array}$ & $\frac{\mathfrak{d}}{\stackrel{\mathcal{S}}{\mathcal{S}}}$ & 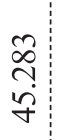 & 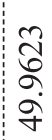 \\
\hline 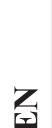 & 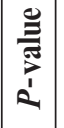 & \begin{tabular}{c:c}
0 & \\
\hdashline & 1 \\
& \\
&
\end{tabular} & $\begin{array}{l}0 \\
0 \\
\sim \\
\sim\end{array}$ & $\begin{array}{c}0 \\
d \\
d \\
\end{array}$ & $\begin{array}{l}0 \\
\mathfrak{d} \\
\sim \\
\sim\end{array}$ & $\begin{array}{c}0 \\
j \\
\sim \\
\sim\end{array}$ & 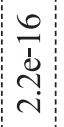 & $\begin{array}{c}0 \\
\\
\mathrm{~d} \\
\mathrm{~d}\end{array}$ & 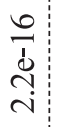 & $\begin{array}{l}o \\
d \\
d \\
d\end{array}$ & $\begin{array}{c:}o \\
\\
\end{array}$ & 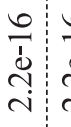 & 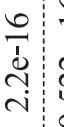 & 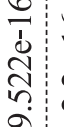 & $\begin{array}{l:}o \\
\\
\end{array}$ & $\begin{array}{c}0 \\
\\
\\
\text { d }\end{array}$ & 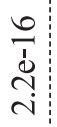 & $\begin{array}{l}\vec{J} \\
\dot{d} \\
o \\
\sim\end{array}$ & $\begin{array}{l}0 \\
d \\
\mathrm{~d} \\
\mathrm{~d}\end{array}$ & $\begin{array}{c:c}0 \\
\\
\\
\\
\end{array}$ & 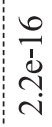 \\
\hline $\mathbb{1}$ & 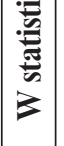 & \begin{tabular}{l}
$\stackrel{8}{0}$ \\
0 \\
\hdashline \\
0
\end{tabular} & 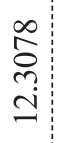 & $\begin{array}{l}\tilde{r} \\
\stackrel{\sigma}{-}\end{array}$ & 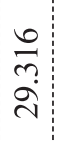 & 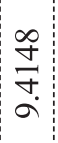 & \begin{tabular}{c}
0 \\
\multirow{2}{*}{} \\
$\mathrm{I}$
\end{tabular} & $\overrightarrow{\hat{n}}$ & 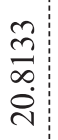 & $\begin{array}{l}\infty \\
\infty \\
n \\
\infty\end{array}$ & $\begin{array}{l}\infty \\
\infty \\
\infty \\
\dot{\lambda}\end{array}$ & 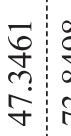 & 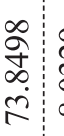 & 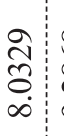 & $\begin{array}{l}\infty \\
\infty \\
\infty \\
\\
\alpha\end{array}$ & $\begin{array}{l}\hat{\sigma} \\
\stackrel{0}{\varrho}\end{array}$ & 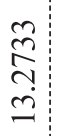 & $\begin{array}{l}\underset{J}{J} \\
\stackrel{+}{\sim}\end{array}$ & 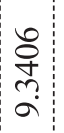 & $\begin{array}{l}\sqrt[n]{0} \\
\stackrel{2}{\Xi} \\
=\end{array}$ & $\begin{array}{l}\infty \\
0 \\
n \\
n \\
n\end{array}$ \\
\hline 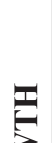 & 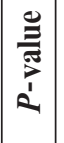 & $\begin{array}{l}\infty \\
\hat{n} \\
\infty \\
0\end{array}$ & $\begin{array}{c:}0 \\
d \\
\sim \\
\sim\end{array}$ & $\begin{array}{c}0 \\
d \\
d \\
\end{array}$ & $\begin{array}{c:}0 \\
d \\
\sim \\
\sim\end{array}$ & $\begin{array}{l}\frac{n}{d} \\
\frac{\sigma}{2} \\
m\end{array}$ & 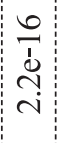 & $\begin{array}{l}\infty \\
\infty \\
\\
\\
0\end{array}$ & $\begin{array}{l:}\stackrel{0}{0} \\
\text { กิ }\end{array}$ & 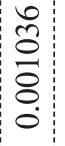 & $\begin{array}{l}\infty \\
0 \\
d \\
\stackrel{2}{d} \\
\stackrel{n}{n}\end{array}$ & 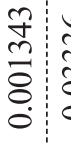 & 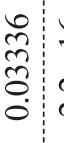 & 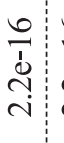 & $\begin{array}{l:}\stackrel{0}{1} \\
\end{array}$ & $\begin{array}{l}\frac{D}{d} \\
\delta \\
\delta \\
d\end{array}$ & 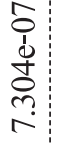 & $\begin{array}{l}0 \\
\dot{d} \\
\stackrel{\sim}{\sim}\end{array}$ & $\begin{array}{l}0 \\
d \\
\mathrm{~d} \\
\mathrm{v}\end{array}$ & $\begin{array}{c:c}0 & \\
& \\
& \\
& \end{array}$ & 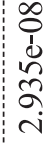 \\
\hline ज) & 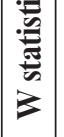 & 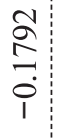 & 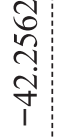 & 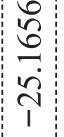 & $\frac{\sigma}{\stackrel{J}{S}}$ & $\begin{array}{l}\tilde{N} \\
n \\
\infty\end{array}$ & $\begin{array}{l}\bar{n} \\
\tilde{\infty} \\
\sigma\end{array}$ & $\begin{array}{l}\infty \\
\infty \\
\infty\end{array}$ & 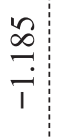 & $\begin{array}{l}0 \\
\infty \\
1 \\
n\end{array}$ & $\begin{array}{l}m \\
n \\
n\end{array}$ & 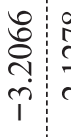 & $\begin{array}{l:l}\infty & \vdots \\
& \vdots \\
& s\end{array}$ & 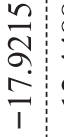 & $\begin{array}{l}\infty \\
+\infty \\
\stackrel{+}{+} \\
\stackrel{1}{1}\end{array}$ & 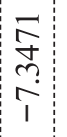 & $\begin{array}{l}\vec{n} \\
\tilde{n} \\
\dot{+}\end{array}$ & \begin{tabular}{l}
$\infty$ \\
$\stackrel{\infty}{n}$ \\
$\stackrel{2}{n}$ \\
\hdashline \\
1
\end{tabular} & $\begin{array}{l}\stackrel{2}{\partial} \\
\infty \\
\frac{\infty}{1}\end{array}$ & $\begin{array}{c:}\vec{J} \\
\vec{\Delta} \\
\infty \\
1\end{array}$ & $\begin{array}{l}n \\
n \\
\tilde{f} \\
n \\
i\end{array}$ \\
\hline 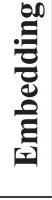 & 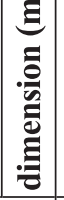 & $N$ & $m$ & $\nabla$ & $n$ & $N$ & $m$ & $\nabla$ & $n$ & $\sim$ & $m$ & $\nabla 4$ & $n$ & $N$ & $m$ & $\nabla$ & $n$ & $N$ & $m$ & $\nabla$ & in \\
\hline \multicolumn{2}{|c|}{$\underset{\Xi}{\Xi}$} & \multicolumn{4}{|c|}{ 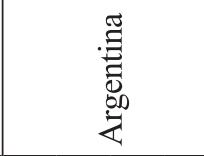 } & \multicolumn{4}{|c|}{ 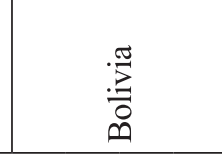 } & \multicolumn{4}{|c|}{ 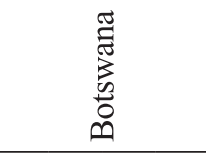 } & \multicolumn{4}{|c|}{$\begin{array}{l}\overline{\mathbb{N}} \\
\text { ص్ }\end{array}$} & \multicolumn{4}{|c|}{$\frac{0}{\tilde{\theta}}$} \\
\hline
\end{tabular}




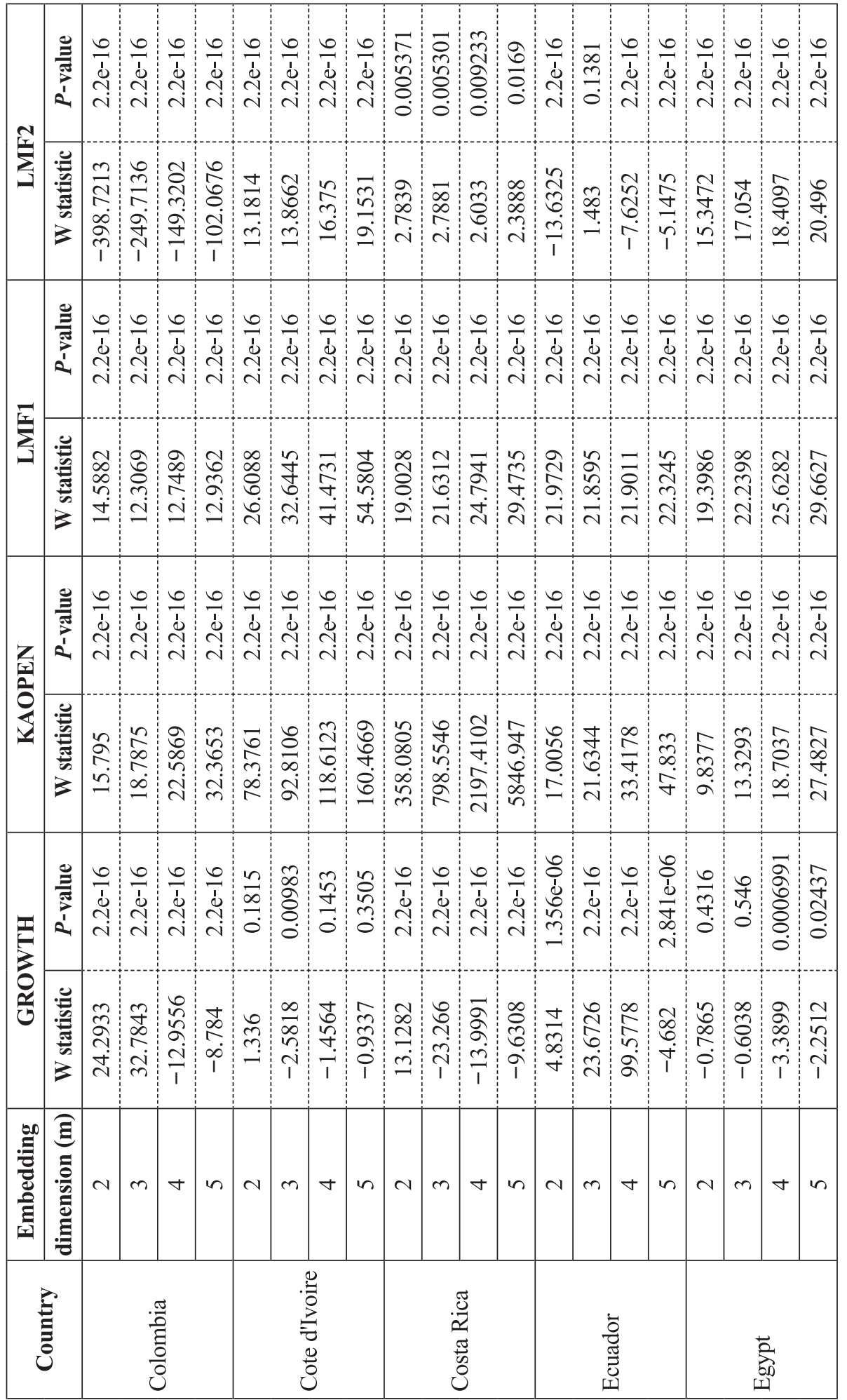




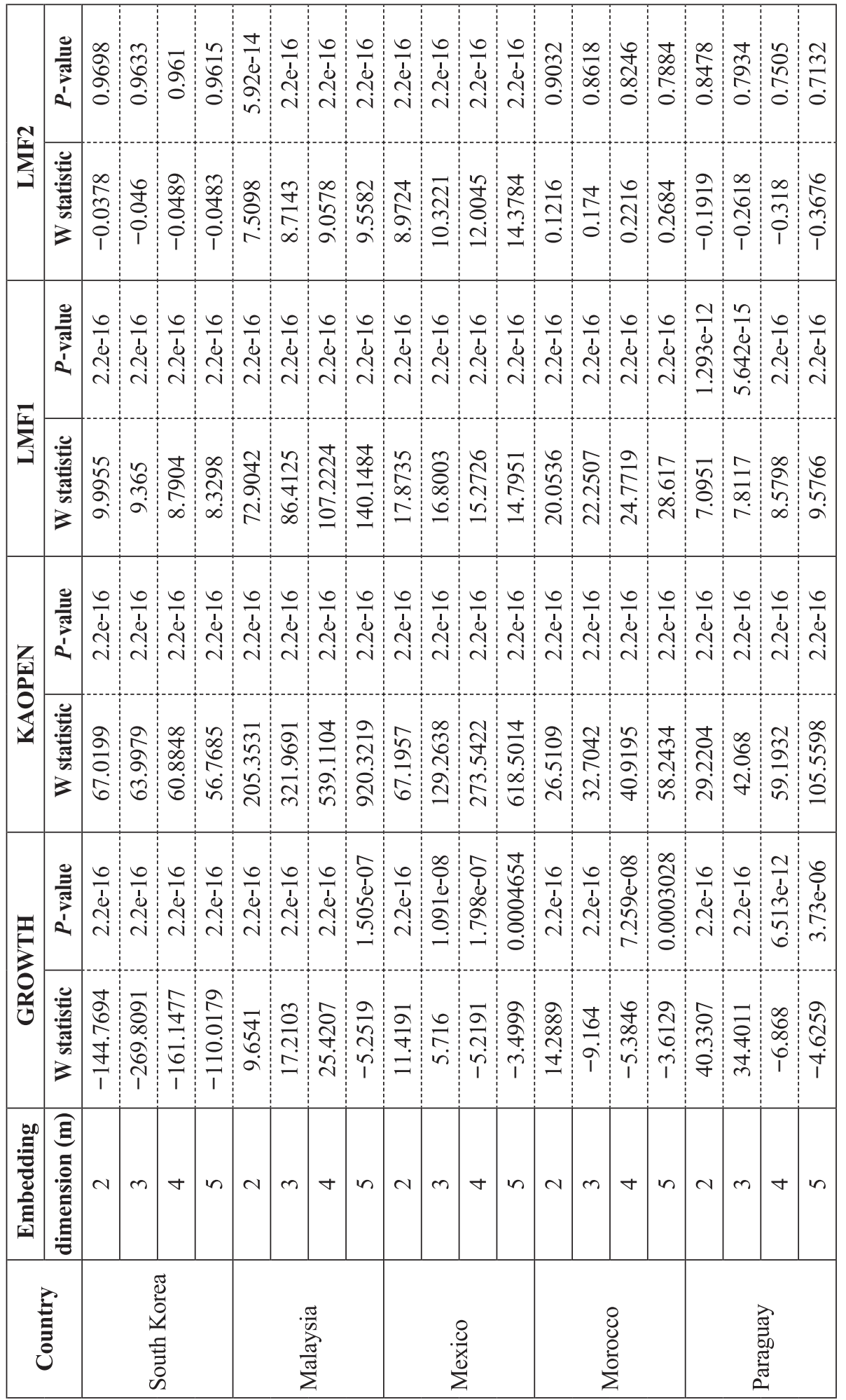




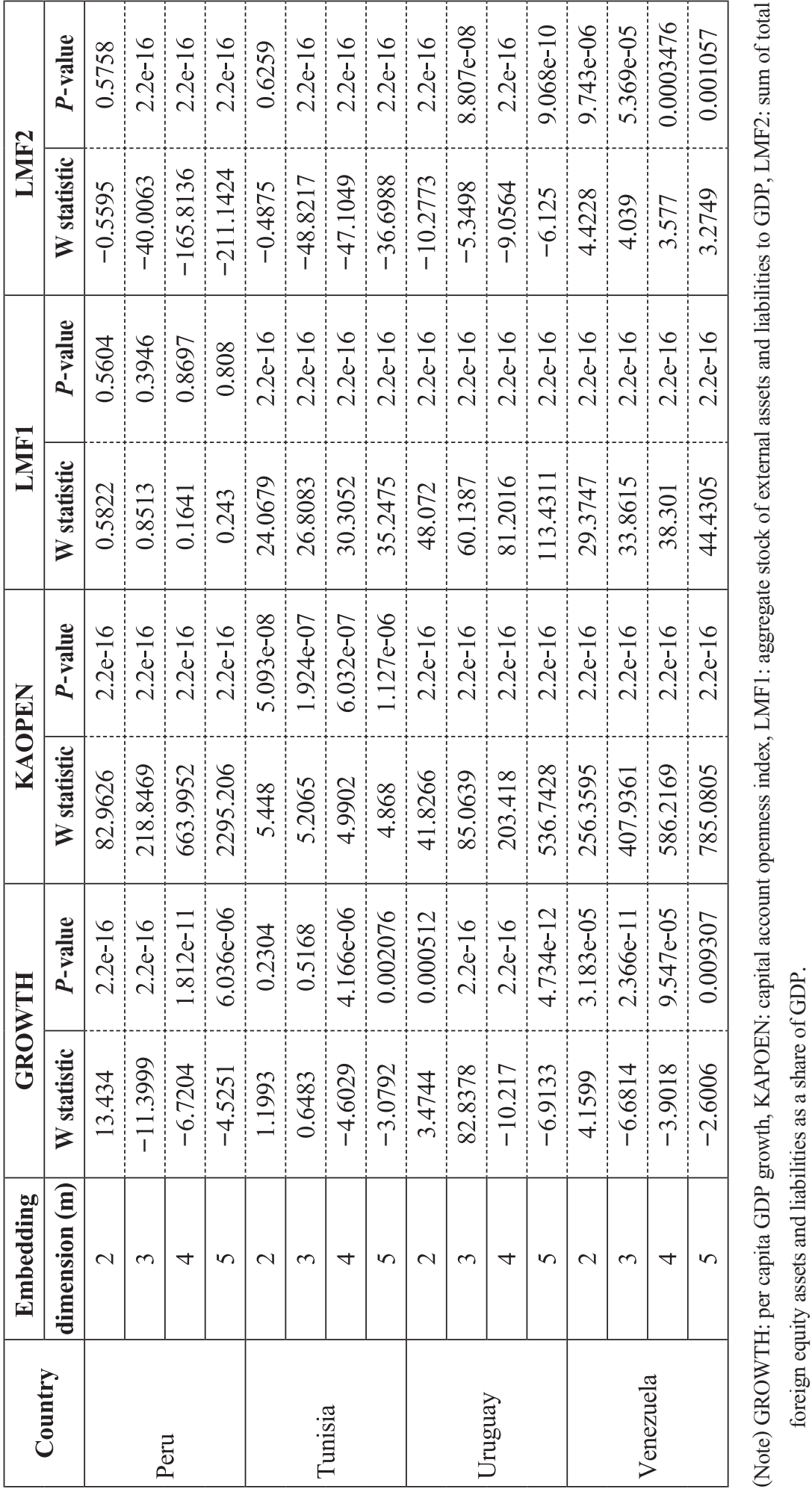


Table 6. Granger (non-)causality $S_{F}$ test statistics

\begin{tabular}{|l|c|c|c|c|c|c|}
\hline \multirow{2}{*}{ Country } & \multicolumn{3}{|c|}{$\begin{array}{r}\text { Financial integration } \rightarrow \\
\text { Economic growth }\end{array}$} & \multicolumn{3}{c|}{$\begin{array}{c}\text { Economic growth } \\
\text { Financial integration }\end{array}$} \\
\cline { 2 - 7 } & KAOPEN & LMF1 & LMF2 & KAOPEN & LMF1 & LMF2 \\
\hline Argentina & $5.58^{* * *}$ & $7.43^{* * *}$ & 1.42 & $0.0549^{* * *}$ & 0.119 & 0.21 \\
\hline Bolivia & 5.12 & $2.53^{*}$ & $3.44^{* *}$ & 0.454 & $5.05^{* * *}$ & 0.507 \\
\hline Botswana & 0.135 & 0.0774 & 0.0774 & 0.0348 & $3.35^{*}$ & $3.35^{*}$ \\
\hline Brazil & 0.296 & 0.257 & 1.25 & 0.0215 & $4.15^{* * *}$ & $3.12^{* *}$ \\
\hline Chile & 0.0143 & 0.309 & 2.61 & $4.13^{* *}$ & 0.52 & $4 * *$ \\
\hline Cote d'Ivoire & 0.476 & 0.0738 & 1.2 & $12 * * *$ & $2.7 *$ & $5.31^{* *}$ \\
\hline Colombia & 1.38 & $3.55^{*}$ & $6.85^{* *}$ & 0.0331 & 0.118 & 0.0166 \\
\hline Costa Rica & 0.585 & 0.363 & 1.65 & 0.183 & $5.85^{* * *}$ & $2.43^{*}$ \\
\hline Ecuador & 0.362 & 0.0887 & 0.148 & 0.0253 & $14.5^{* * *}$ & $22.6^{* * *}$ \\
\hline Egypt & 0.0887 & 0.0999 & 0.596 & $14.5^{* * *}$ & $3.73^{* *}$ & 0.243 \\
\hline South Korea & 0.0426 & 0.0603 & 0.154 & 0.112 & $4.98^{* * *}$ & $5.14 * * *$ \\
\hline Malaysia & 0.376 & 0.0512 & 0.072 & $3.26^{* *}$ & $2.56^{*}$ & $11.4^{* * *}$ \\
\hline Mexico & 0.409 & 0.435 & 0.0103 & $2.96^{*}$ & $5.54^{* *}$ & 0.227 \\
\hline Morocco & 0.44 & $11 * * *$ & $9.58^{* * *}$ & 0.254 & 0.0635 & 1.24 \\
\hline Paraguay & 0.0567 & 1.02 & 0.0567 & $12.2^{* * *}$ & 0.296 & $12.2 * * *$ \\
\hline Peru & 0.0208 & 0.353 & 0.0168 & 2.16 & 0.404 & 0.581 \\
\hline Tunisia & 1.82 & $2.11 *$ & $3.58^{* *}$ & 0.561 & 0.128 & 0.45 \\
\hline Uruguay & $7.8 * * *$ & 0.0233 & $3.69^{* *}$ & $2.79^{* * *}$ & 0.374 & 0.126 \\
\hline Venezuela & $8.63 * * *$ & $3.35^{* *}$ & 2.53 & 0.0965 & 1.08 & 0.694 \\
\hline
\end{tabular}

(Notes) (i) GROWTH: per capita GDP growth, KAPOEN: capital account openness index, LMF1: aggregate stock of external assets and liabilities to GDP, LMF2: sum of total foreign equity assets and liabilities as a share of GDP.

(ii) For $\mathrm{X} \rightarrow \mathrm{Y}, \mathrm{H} 0$ : $\mathrm{X}$ does not cause $\mathrm{Y}$.

(iii) $* * *, * *$ and $*$ denote rejection of the null hypothesis at the $1 \%, 5 \%$, and $10 \%$ significance levels, respectively. 


\section{Conclusions}

The main goal of this study was to investigate the linear and nonlinear causal linkages between financial integration and economic growth in 19 emerging and developing countries over the 1970 2011 period. To that end, we applied both linear and nonlinear causality tests to examine those relations. In particular, apart from the implementation of the modified version of the Granger causality test based on Toda and Yamamoto (1995), we employed the nonlinear and asymmetric causality test of Kyrtsou and Labys (2006), which, unlike the conventional Granger causality test, has the ability to detect nonlinear causal relations between variables. Overall, the findings obtained from the nonlinear causality test tend to reject the neutrality hypothesis for the financial integration-growth relation in 18 of the 19 developing countries under consideration. In the majority of the countries under investigation, the evidence is in line with the growth hypothesis where causality running from economic growth to financial integration was detected in Brazil, Chile, Cote d'Ivoire, Costa Rica, Ecuador, Egypt, South Korea, Malaysia, Mexico, and Paraguay. The opposite causality running from financial integration to economic growth was found in Argentina, Bolivia, Colombia, Morocco, Tunisia, and Venezuela. In contrast, the neutrality hypothesis was supported only in Peru, whereas the feedback hypothesis was supported in Bolivia and Uruguay.

The above heterogeneity in the causality results may suggest that there are important differences in the mechanisms through which financial integration affects economic growth across countries. These mechanisms include financial sector development (Hermes and Lensink 2003, Alfaro et al. 2004, Kose et al. 2011), institutional quality (Bekaert et al. 2005, Chanda 2005), trade openness (Eichengreen 2001, Aizenman and Noy 2008), stability of macroeconomic policies (Arteta et al. 2003, Mody and Murshid 2005, Chen and Quang 2014) and the sectoral composition of the economy (Guven 2016), among others. Other factors, such as domestic credit (Lane and McQuade 2014), fiscal policy (Pierdzioch 2004, Koenig and Zeyneloglu 2010), and initial levels of economic development (Vo and Daly 2007), may also determine the degree of financial integration and, in turn, impact the strength and causality direction of the financial integration-growth nexus.

Some highlights can be drawn from the evidence presented in this study. First, the causal relation between financial integration and economic growth is not uniform across the emerging and developing countries. Therefore, the study confirms that the 
homogeneity assumption in previous studies (Kose et al. 2011, Chen and Quang 2014), even for developing countries, can result in misleading findings while analyzing the link between financial integration and economic growth. In addition, the link between financial integration and economic growth is sensitive to the indicator of financial integration. Taken together, the results of this study indicate clear evidence of the nonlinear causality relation between these two variables. The neutrality hypothesis seems to be rejected for the majority of the 19 developing countries studied during the 1970 2011 period.

Furthermore, regarding the empirical approach, the findings also highlight the importance of testing for nonlinear linkages in addition to linear ones. We found that while the linear causality test indicated that there is no causality between economic growth and each of the financial integration indicators in either direction in Argentina, Botswana, Brazil, Egypt, and Morocco, there was evidence of nonlinear Granger causality for all five countries. Therefore, the existence of a dynamic nonlinear relationship between the two variables was established. In this respect, these results may be useful in future work, as they suggest that researchers should consider nonlinear empirical regularities when exploring the relationship between financial integration and economic growth.

In terms of policy implications, the results here suggest that in countries where bidirectional Granger causality between financial integration and economic growth was found, policies designed to enhance financial integration and economic growth will be mutually beneficial. In countries where evidence shows unidirectional Granger causality running from financial integration to economic growth, policies formulated to promote financial integration will lead to increased economic growth. Moreover, policies designed to enhance efficiency of the financial system could possibly lead to an increase in economic growth. However, in a country where Granger causality runs from economic growth to financial integration, policies designed to enhance growth will promote financial integration.

Despite our promising results, this study suffers from several limitations. First, the bivariate framework used here may be subject to the problem of potential omitted variable bias. Thus, this framework can be readily extended to other multivariate modeling frameworks, where financial integration and economic growth are also determined by other economic factors such as initial levels of economic development, financial sector development, trade openness, and the quality of state institutions. Such an analysis helps disentangle the channels through which financial openness affects economic growth (and vice versa). Second, even if our testing procedure can detect nonlinear causal dependence 
with high power, it provides no guidance regarding the source of the nonlinear dependence. Such guidance seems to be an area for potentially fruitful future research. In addition, it is possible that the causal relation between variables of interest changes over time. In such cases, time-varying causality tests represent an attractive alternative.

Received 20 April 2016, Revised 20 April 2016, Accepted 25 October 2016

\section{References}

Abu-Bader, Suleiman, and Abu-Qarn, Aamer. "Financial development and economic growth: empirical evidence from MENA countries." Review of Development Economics 12 (2008): 803-817.

Acemoglu, Daron, and Zilibotti, Fabrizio. "Was Prometheus unbound by chance? Risk Diversification, and Growth."Journal of Political Economy 105 (1997): 709-751.

Ahmed, Abdullahi D. "International financial integration, investment and economic performance in sub-Saharan African countries." Global Economy Journal 4, 5 (2011): $1-26$.

Ahmed, Abdullahi D. "Effects of financial liberalization on financial market development and economic performance of the SSA region: an empirical assessment." Economic Modelling, 30 (2013): 261-273.

Ahmed, Abdullahi D."Integration of financial markets, financial development and growth: Is Africa different?"Journal of International Financial Markets, Institutions \& Money, 42 (2016): 43-59.

Ahmed, Abdullahi D., and Mmolainyane, Kelesego K. "Financial integration, capital market development and economic performance: empirical evidence from Botswana." Economic Modelling 42 (2014): 1 - 14.

Ajmi, Ahdi N., El Montasser, Ghassen, and Nguyen, Duc K. "Testing the relationships between energy consumption and income in $\mathrm{G} 7$ countries with nonlinear causality tests." Economic Modelling 35 (2013): 126 - 133. 
Alfaro, Laura, Chanda, Areendam, Kalemli, Ozcan S., and Sayek, Selin. "FDI and economic growth: the role of local financial markets", Journal of International Economics 64 (2004): 89-112.

Alfaro, Laura, Kalemli, Ozcan S., and Volosovych, Vadym. "Capital flows in a globalised world: the role of policies and institutions." InNBER Working Paper, No. 11696, National Bureau of Economic Research, Cambridge, Massachusetts, 2005.

Amiri, Arshia, and Ventelou, Bruno. "Granger causality between total expenditure on health and GDP in OECD: Evidence from the Toda-Yamamoto approach." Economics Letters 116 (2012): 541-544.

Arteta, Carlos, Eichengreen, Barry, and Wyplosz, Charles. "When does capital account liberalization help more than it hurts?" In: Helpman, E., Sadka, E. (Eds.), Economic Policy in the International Economy: Essays in Honor of Assaf Razin, pp.177-206. Cambridge: University Press, Cambridge, 2003.

Atil, Ahmed, Lahiani, Amine, and Nguyen, Duc K. "Asymmetric and nonlinear passthrough of crude oil prices to gasoline and natural gas prices", Energy Policy 65 (2014): $567-573$.

Aizenman, Joshua, and Noy, Ilan. "Links between trade and finance - a disaggregated analysis." In Edwards, S., Garcia, M.G.P. (Eds.), Financial Markets Volatility and Performance in Emerging Markets, National Bureau of Economic Research Conference Report. University of Chicago Press, Chicago, 2008.

Baek, Khung G, and Brock, William A. "A General Test for Non-Linear Granger Causality: Bivariate Model." Working Paper, Iowa State University and University of Wisconsin, Madison, WI, 1992.

Baltagi, Badi H., Demetriades, Panicos O., and Law, Siong H. "Financial development and openness: evidence from panel data." Journal of Development Economics 89 (2009): 285-296.

Bekaert, Geert, Harvey, Campbell R., and Lundblad, Christian. "Does financial liberalization spur growth?" Journal of Financial Economics 77, 1 (2005): 3-55.

Bildirici, Melike E., and Turkmen, Ceren. "Nonlinear causality between oil and precious metals." Resources Policy 46 (2015): 202-211. 
Bonfiglioli, Alessandra. "Financial integration, productivity and capital accumulation." Journal of International Economics 76 (2008): 337-355.

Brock, William, Dechect, Davis W., and Scheinkman, José A. “A test for independence based on the correlation dimension." Working Paper, Department of Economics, University of Wisconsin, Madison, 1987.

Bumann, Silke, Hermes, Niels, and Lensink, Robert. "Financial liberalization and economic growth: A meta-analysis." Journal of International Money and Finance 33 (2013): 255-281.

Bussière, Matthieu, and Fratzscher, Marcel. "Financial openness and growth: short run gain, long-run pain." Review of International Economics 16, 1 (2008): 69-95.

Chanda, Areendam. "The influence of capital controls on long run growth: where and how much?” Journal of Development Economics 77, 2 (2005): 441-466.

Chen, Jinzhao, and Quang, Thérèse. "The impact of international financial integration on economic growth: New evidence on threshold effects." Economic Modelling 42 (2014): 475-489.

Chinn, Menzie D., and Ito, Hiro. "A new measure of financial openness.”Journal of Comparative Policy Analysis 10, 3 (2008): 309-322.

Clark,Judith A., and Mirza, Sadaf. "Comparison of some common methods of detecting Granger noncausality." Journal of Statistical Computation and Simulation 76 (2006): $207-231$.

De Nicolò, Gianni, and Juvenal, Luciana. "Financial integration, globalization, and real activity." Journal of financial stability 10 (2014): 65-75.

Dickey, David A., and Fuller, Wayne A. "Distribution of The Estimators for Autoregressive Time Series with a Unit Root." Journal of the American Statistical Association 74 (1979): $427-431$.

Dinar, Gülenay B., Dalgiç,Başak C., and Yidogan, Pelin V. "Financial Liberalization and Economic Growth in Turkey: A Reexamination." Journal of Economics and Administrative Sciences 33, 1 (2015): 19-43.

Dolado, Juan J., and Lütkepohl, Helmut. "Making Wald Test Work for Cointegrated VAR Systems.” Econometric Theory 15 (1996): 369-386. 
Edison, Hali J., Levine, Ross, Ricci, Luca, and Sløk, Torsten. "International financial integration and economic growth." Journal of International Money and Finance 21, 6 (2002): 749-776.

Eichengreen, Barry. "Capital account liberalization: what do cross-country studies tell us?" World Bank Economic Review 15, 3 (2001): 341-365.

Eichengreen, Barry, and Leblang, David. "Capital account liberalization and growth: was Mr. Mahathir right?" International Journal of Finance and Economics 8 (2003): 205-224.

Fratzscher, Marcel, and Bussière, Matthieu. "Financial openness and growth: short-run gain, long-run gain?” European Central Bank Working Paper, 348 (2004): 1-37.

Friedrich, Christian, Schnabel, Isabel, and Zettelmeyer, Jeromin. "Financial integration and growth-Why is emerging Europe different?"'Journal of International Economics 89 (2013): 522-538.

Gehringer, Agnieszka. "Financial liberalization, growth, productivity and capital accumulation: the case of European integration."Discussion Papers, Center for European Governance and Economic Development Research, nº 86 (2012).

Gehringer, Agnieszka. "Uneven effects of financial liberalization on productivity growth in the EU: Evidence from a dynamic panel investigation." International Journal of Production Economics 159 (2015): 334-346.

Giannetti, Mariassunta, Guiso, Luigi, Jappelli, Tullio, Padula, Mario, and Pagano, Marco. "Financial market integration, corporate financing and economic growth." European commission economic papers, $\mathrm{n}^{\circ} 179$ (2002).

Giles, David E.A. "Causality between the measured and underground economies in New Zealand." Applied Economics Letters 4 (1997): 63-67.

Gourinchas, Pierre O., and Jeanne, Olivier. "Capital flows to developing countries: the allocative puzzle." The Review of Economic Studies 80, 4 (2013): 1484-1515.

Granger, Clive W.J. "Investigating causal relations by econometric models and crossspectral methods." Econometrica 37 (1969): 424-439.

Gregory, AllanW., and Hansen, Bruce E. "Residual-based tests for cointegration in models with regime shifts.", Journal of Econometrics 70 (1996): 99-126. 
Grilli, Vittorio, and Milesi-Ferretti, Gian M. "Economic effects and structural determinants of capital controls.” International Monetary Fund Staff Papers 42, 3 (1995): 517-551.

Guven, Cahit. "Financial integration: The role of tradable and non-tradable goods." Economic Modelling 53 (2016): 345-353.

Henry, Peter B. “Do stock market liberalizations cause investment booms?” Journal of Financial Economics, 58 (2000): 301-334.

Hermes, Niels, and Lensink, Robert. "Foreign direct investment, financial development and economic growth." Journal of Development Studies 40, 1 (2003): 142-163.

Hiemstra, Craig, and Jones, Jonathan D. "Testing for Linear and Nonlinear Granger Causality in the Stock Price Volume Relation.” Journal of Finance 49 (1994): 16391664.

Honig, Adam. "Addressing causality in the effect of capital account liberalization on growth.” Journal of Macroeconomics 30, 4 (2008):1602-1616.

Hristu-Varsekelis, Dimitrios, and Kyrtsou, Catherine. "Evidence for Nonlinear Asymmetric Causality in US Inflation, Metal, and Stock Returns." Discrete Dynamics in Nature and Society 5, 3 (2008):1-7.

Hye, Qazi M. A., and Wizarat, Shahida. "Impact of financial liberalization on economic growth: a case study of Pakistan." Asian Economic and Financial Review 3, 2(2013): 270-282.

Jawaid, Syed T., and Raza, Syed A. "Effect of terms of trade on growth performance in India." Economic Modelling 33 (2013): 940-946.

Johansen, Søren. "Statistical Analysis of Cointegrating Vectors." Journal of Economic Dynamics and Control 12 (1988) 23: 1-254.

Johansen, Søren, and Juselius, Katariana. "Maximum Likelihood Estimation and Inference on Cointegration with Applications to the Demand for Money." Oxford Bulletin of Economics and Statistics 52 (1990): 169-209.

Kemba, Bernd, and Khan, Nazmus S. "Government debt and economic growth in the G7 countries: are there any causal linkages?" Applied Economics Letters 23, 6 (2016): 440443.

Klein, Michael W., and Olivei, Giovanni P. "Capital Account Liberalization, Financial 
Depth, and Economic Growth." updated version of NBER Working Paper, No. 7384 (2001).

Klein, Michael W., and Olivei, Giovanni P. "Capital account liberalization, financial depth, and economic growth." Journal of International Money and Finance 27 (2008): 861-875.

Koenig, Gilbert, and Zeyneloglu, Irem. "When does financial integration matter for fiscal policy in a currency union? A welfare-based approach.” Economic Modelling 27 (2010): 620-630.

Kose, M. Ayhan, Prasad, Eswar, Rogoff,Kenneth, and Wei,Shang-Jin. "Financial globalization: A reappraisal.” IMF Staff Papers 56 (2009): 8-62.

Kose, M. Ayhan, Prasad, Eswar, Rogoff, Kenneth, and Wei, Shang-Jin. "Financial globalization and economic policies", In: Rodrik, D., Rosenzweig, M.R. (Eds.), Handbook of Development Economics, Vol. 5 (2010): 4283-4362. North-Holland, Amsterdam.

Kose, M. Ayhan, Prasad, Eswar, and Taylor, Ashley D. "Thresholds in the process of international financial integration." Journal of International Money and Finance 30, 1 (2011): 147-179.

Kraay, Aart. "In search of the macroeconomic effects of capital account liberalization." Manuscript, World Bank, Washington, DC,1998.

Kumar, Manish. "A bivariate linear and nonlinear causality between stock prices and exchange rates." Economics Bulletin 29, 4 (2009), 2884 - 2895.

Kumar, Manish, and Thenmozhi, M. "Causal effect of volume on stock returns and conditional volatility in developed and emerging market." American Journal of Finance and Accounting 2, 4 (2012): 346-362.

Kwiatkowski, Denis, Phillips, Peter C.B., Schmidt, Peter, and Shin, Yongcheol . "Testing the Null Hypothesis of Stationarity against the Alternative of a Unit Root." Journal of Econometrics 54 (1992): 159-178.

Kyrtsou, Catherine, and Labys, Walter C. "Evidence for chaotic dependence between us inflation and commodity prices.” Journal of Macroeconomics 28 (2006): 256-266.

Lane, Philip R., and McQuade, Peter. "Domestic Credit Growth and International Capital Flows.” The Scandinavian Journal of Economics 116 (2014): 218-252. 
Lane, Philip R., and Milesi-Ferretti, Gian M. "The external wealth of nations Mark II: revised and extended estimates of foreign assets and liabilities, 1970 2004." Journal of International Economics 73, 2 (2007): 223-250.

Le, Thai H., and Chang, Youngho. "Effects of oil price shocks on the stock market performance: Do nature of shocks and economies matter?" Energy Economics 51 (2015): 261-274.

Levine, Ross. "International Financial Liberalization and Economic Growth."Review of International Economics 9 (2001): 668-702.

Malik, Samreen. "Financial-integration thresholds for consumption risk-sharing." International Review of Economics and Finance 38 (2015): 73-93.

Mavrotas, George, and Kelly, Roger. "Old wine in new bottles: testing causality between savings and growth.” The Manchester School 69 (2001): 97-105.

Menyah, Kojo, and Wolde-Rufael, Yemane. "Energy consumption, pollutant emissions and economic growth in South Africa." Energy Economics 32 (2010): 1374-1382.

Mishkin, Frederic S. The next great globalization: How disadvantaged nations can harness their financial systems to get rich, Princeton: Princeton University Press, 2006.

Mmolainyane, Kelesego K., and Ahmed, Abdullahi D. "The impact of financial integration in Botswana."Journal of Policy Modeling 37 (2015): 852-874.

Mody, Ashoka, and Murshid, Antu P. "Growing up with capital flows." Journal of International Economics 65, 1 (2005): 249-266.

Motelle, Sephooko, and Biekpe, Nicholas. "Financial integration and stability in the Southern African development community." Journal of Economics and Business 79 (2015): 100-117.

Nazlioglu, Saban, and Soytas, Ugur. "World oil prices and agricultural commodity prices: evidence from an emerging market."Energy Economics 33 (2011): 488-496.

Obstfeld, Maurice. "Risk-taking, Global Diversification, and Growth." American Economic Review 84 (1994): 1310-1329.

Payne, James E. "On the dynamics of energy consumption in the US." Applied Energy 86 (2009): 575-577. 
Phillips, Peter C.B., and Perron, Pierre. "Testing for Unit root in Time Series Regression." Biometrika 75 (1988): 335-346.

Pierdzioch, Christian. "Capital mobility and the effectiveness of fiscal policy in open economies." Journal of Macroeconomics 26 (2004): 465-479.

Prasad, Eswar, Rogoff, Kenneth, Wei, Shang J. and Kose, M.Ayhan. "Effects of financial globalization on developing countries: some empirical evidence." Occasional Paper, $\mathrm{n}^{\circ}$ 220, International Monetary Fund, Washington DC (2003).

Quinn, Dennis P. "The correlates of change in international financial regulation." American Political Science Review 91, 3 (1997): 531-551.

Quinn, Dennis P., and Toyoda, A. Maria."Does capital account liberalization lead to growth?" The Review of Financial Studies 21, 3 (2008): 1403-1449.

Rajan, Raghuram G., and Zingales, Luigi. "Financial dependence and growth." American Economic Review 88 (1998): 559-586.

Rambaldi, Alicia N., and Doran, Howard E. "Testing for Granger non-causality in cointegrated system made easy." Working Papers in Econometrics and Applied Statistics, No. 88, Department of Econometrics, University of New England (1996).

Ray, Sarbapriya. "Causal linkage between international financial integration and economic growth: evidence from post globalized Indian scenario." Advances in Asian Social Science 3, 4 (2012): 739-750.

Rodrik, Dani. "Who Needs Capital-Account Convertibility?" In: Fischer, S. (Ed.), Should the IMF Pursue Capital Account Convertibility? Essays in International Finance, No. 207 International Finance Section, Department of Economics, Princeton University, Princeton, New Jersey, 1998.

Saafi, Sami, Farhat,Abdeljelil, and Mohamed, Meriem B.H. "Testing the relationships between shadow economy and unemployment: empirical evidence from linear and nonlinear tests." Studies in Nonlinear Dynamics \& Econometrics 19, 5 (2015a): 585-608.

Saafi, Sami, Mohamed, Meriem B.H., and Farhat,Abdeljelil. "Is there a causal relationship between unemployment and informal economy in Tunisia: evidence from linear and non-linear Granger causality."Economics Bulletin 35, 2 (2015b): 1191-1204.

Sandri, Damiano. "Growth and capital flows with risky entrepreneurship." American 
Economic Journal: Macroeconomics 6, 3 (2010): 102-123.

Schularick, Moritz, and Steger, Thomas M. "Financial integration, investment, and economic growth: evidence from two eras of financial globalization." The Review of Economics and Statistics 92, 4 (2010): 756-768.

Sims, Christopher A., Stock, James H., and Watson, Mark W. "Inference in Linear Time Series Models with Unit Roots." Econometrica 58 (1990): 113-144.

Toda, Hiro Y., and Phillips, Peter C.B. "Vector Autoregressions and Causality." Econometrica 61 (1993): 1367-1393.

Toda, Hiro Y. and Yamamoto, Taku. "Statistical Inference in Vector Autoregressions with Possibly Integrated Processes.” Journal of Econometrics 66 (1995): 225-250.

Vithessonthi, Chaipor, and Tongurai, Jittima. "The impact of capital account liberalization measures." Journal of International Financial Markets, Institutions and Money 22 (2012): 16-34.

Vo, Xuan V., and Daly, Kevin J. "The determinants of international financial integration." Global Finance Journal 18 (2007): 228-250.

Wolde-Rufael, Yemane. "Re-examining the financial development and economic growth nexus in Kenya." Economic Modelling 26, 6 (2009): 1140-1146.

Zapata, Hector O., and Rambaldi,Alicia N. "Monte Carlo evidence on cointegration and causation." Oxford Bulletin of Economics and Statistics 9 (1997): 285-298.

Zivot, Eric, and Andrews, Donald W.K. "Further Evidence on the Great Crash, the Oil Price Shock, and the Unit-Root Hypothesis." Journal of Business and Economic Statistics10, 3 (1992): 251-270. 